Commun. Math. Phys. 182, $105-153$ (1996)

\title{
On the Dimension of Deterministic and Random Cantor-like Sets, Symbolic Dynamics, and the Eckmann-Ruelle Conjecture
}

\author{
Yakov Pesin, Howard Weiss
}

Department of Mathematics, 310, McAlister Building, The Pennsylvania State University, University Park, PA 16802, USA. E-mails: pesin@math.psu.edu, weiss@math.psu.edu

Received: 15 November 1995/Accepted: 14 Febrauary 1996

\begin{abstract}
In this paper we unify and extend many of the known results on the dimension of deterministic and random Cantor-like sets in $\mathbb{R}^{n}$, and apply these results to study some problems in dynamical systems. In particular, we verify the Eckmann-Ruelle Conjecture for equilibrium measures for Hölder continuous conformal expanding maps and conformal Axiom $\mathrm{A}^{\#}$ (topologically hyperbolic) homeomorphims. We also construct a Hölder continuous Axiom $\mathrm{A}^{\#}$ homeomorphism of positive topological entropy for which the unique measure of maximal entropy is ergodic and has different upper and lower pointwise dimensions almost everywhere. This example shows that the non-conformal Hölder continuous version of the Eckmann-Ruelle Conjecture is false.

The Cantor-like sets we consider are defined by geometric constructions of different types. The vast majority of geometric constructions studied in the literature are generated by a finite collection of $p$ maps which are either contractions or similarities and are modeled by the full shift on $p$ symbols (or at most a subshift of finite type). In this paper we consider much more general classes of geometric constructions: the placement of the basic sets at each step of the construction can be arbitrary, and they need not be disjoint. Moreover, our constructions are modeled by arbitrary symbolic dynamical systems. The importance of this is to reveal the close and nontrivial relations between the statistical mechanics (and especially the absence of phase transitions) of the symbolic dynamical system underlying the geometric construction and the dimension of its limit set. This has not been previously observed since no phase transitions can occur for subshifts of finite type.

We also consider nonstationary constructions, random constructions (determined by an arbitrary ergodic stationary distribution), and combinations of the above.
\end{abstract}

\section{Introduction}

In this paper we unify and extend many of the known results on the dimension of deterministic and random Cantor-like sets in $\mathbb{R}^{n}$. These sets are defined by geometric

The work of the first author was partially supported by a National Science Foundation grant \#DMS9102887. The work of the second author was partially supported by a National Science Foundation Postdoctoral Research Fellowship and National Science Foundation grant \#DMS-9403724. 
constructions of different types. The vast majority of geometric constructions studied in the literature are generated by a finite collection of $p$ maps which are either contractions or similarities and are modeled by the full shift on $p$ symbols. In this paper we consider much more general classes of geometric constructions: their basic sets may have complicated geometry and topology and the placement of the basic sets may be fairly arbitrary. Moreover, basic sets on a given step of the construction may intersect each other. We also allow geometric constructions modeled by an arbitrary symbolic dynamical system.

Our inspiration for studying these classes of geometric constructions comes from the theory of hyperbolic dynamical systems. For instance, Smale-Williams solenoids are limit sets for geometric constructions which we consider. Moreover, using Markov partitions, any locally maximal hyperbolic set can be viewed as the limit set for some geometric construction. We will also apply our results on geometric constructions to study a Hölder continuous version of the Eckmann-Ruelle conjecture (see Sect. 6). A detailed announcement of this paper appeared in [PW1].

A prototype for our analysis is a geometric construction which defines a Cantorlike set of the form

$$
F=\bigcap_{n=1}^{\infty} \bigcup_{\left(i_{1} \cdots i_{n}\right)} \Delta_{i_{1} \cdots i_{n}}
$$

where the basic sets on the $n^{\text {th }}$ step of the construction, $\Delta_{i_{1} \cdots i_{n}}, i_{k}=1, \ldots, p$, are closed, disjoint, and $\Delta_{l_{1} \cdots i_{n} j} \subset \Delta_{i_{1} \cdots i_{n}}$, for $j=1, \ldots, p$. We assume that the $\operatorname{diam}\left(\Delta_{i_{1} \cdots i_{n}}\right) \rightarrow 0$ as $n \rightarrow \infty$ and we emphasize that the placement of the sets $\Delta_{i_{1} \cdots i_{n}}$ can be arbitrary as long as they satisfy the above conditions. See Fig. 1.

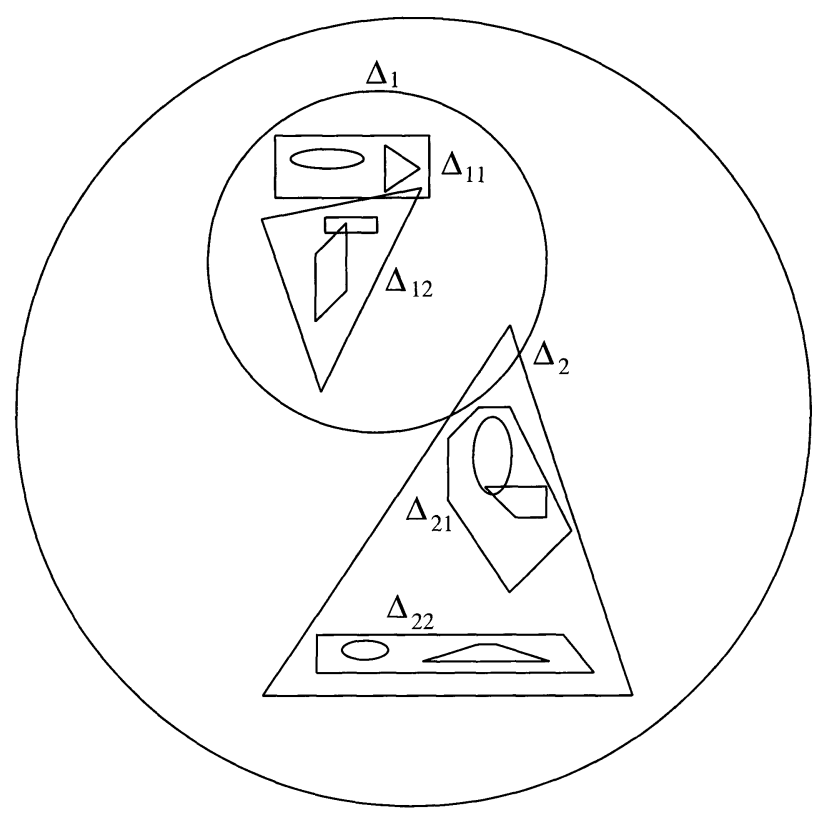

Fig. 1. Simple Geometric Construction 
About 50 years ago, Moran [Mo] computed the Hausdorff dimension of limit sets of geometric constructions in $\mathbb{R}^{n}$ given by $p$ basic sets $\Delta_{i_{1} \cdots i_{n}}$ satisfying

(1) each basic set is the closure of its interior,

(2) at each level the basic sets do not overlap (their interiors are disjoint),

(3) a basic set $\Delta_{i_{1} \cdots i_{n} j}$ is geometrically similar to the basic set $\Delta_{i_{1} \cdots i_{n}}$ for every $j$ and $n$,

(4) $\operatorname{diam}\left(\Delta_{i_{1} \cdots l_{n} j}\right)=\lambda_{j} \operatorname{diam}\left(\Delta_{i_{1} \cdots i_{n}}\right)$, where $0<\lambda_{j}<1$ for $j=1, \ldots, p$ are the ratio coefficients.

These constructions are called Moran constructions. Moran discovered the formula $s=\operatorname{dim}_{H} F$, where $s$ is the unique root of the equation

$$
\sum_{l=1}^{p} \lambda_{i}^{t}=1
$$

and $\operatorname{dim}_{H} F$ is the Hausdorff dimension of the limit set $F$. He also showed that the $s$-dimensional Hausdorff measure of the limit set is finite and strictly positive.

Moran's major idea was to construct an optimal cover of the limit set (Moran cover) which is determined by the ratio coefficients. Our main insight into the Moran approach is that many of the strict conditions in the definition of Moran construction are not required to build the Moran cover. For example, the geometric similarity of basic sets may be greatly weakened. Furthermore, although Moran only considered constructions modeled by the full shift, his approach can be generalized to constructions modeled by arbitrary symbolic dynamical systems. Our approach allows us to extend the original Moran idea to much broader classes of geometric constructions.

In particular, we introduce the Moran-like constructions defined as follows. Let $(Q, \sigma)$ be a symbolic dynamical system where $Q \subset \Sigma_{p}^{+}$is a compact shift invariant subset. We allow basic sets $\Delta_{i_{1} \cdots i_{n}}$ with $n$-tuples $i_{1} \cdots i_{n}$ which are admissible with respect to $Q$ such that

$$
\underline{B}_{i_{1} \cdots i_{n}} \subset \Delta_{i_{1} \cdots l_{n}} \subset \bar{B}_{l_{1} \cdots i_{n}},
$$

where $\underline{B}_{i_{1} \cdots i_{n}}$ and $\bar{B}_{i_{1} \cdots i_{n}}$ are closed balls having radii $\underline{r}_{i_{1} \cdots i_{n}}$ and $\bar{r}_{i_{1} \cdots i_{n}}$ respectively,

(2) int $\underline{B}_{i_{1} \cdots i_{n}} \cap$ int $\underline{B}_{i_{1}^{\prime} \cdots i_{n}^{\prime}}=\emptyset$ if $\left(i_{1} \cdots i_{n}\right) \neq\left(i_{1}^{\prime}, \ldots, i_{n}^{\prime}\right)$,

(3) $\underline{r}_{i_{1} \cdots i_{n}}=C_{1} \prod_{j=1}^{n} \lambda_{i_{j}}$ and $\bar{r}_{i_{1} \cdots i_{n}}=C_{2} \prod_{j=1}^{n} \lambda_{i_{j}}$, where $0<\lambda_{i}<1, i=1, \ldots, p$ and $C_{1}, C_{2}$ are positive constants.

We stress that the topology and geometry of basic sets may be quite complicated. For example, they may not be connected and their boundaries may be fractal. In particular the basic sets at level $n$ of the construction need not be geometrically similar to the basic sets at level $n-1$. Furthermore the basic sets at a given level may intersect. This class of constructions includes Moran geometric constructions.

One of our main results establishes the coincidence of the Hausdorff dimension and the box dimension of the limit sets of these Moran-like constructions, along with explicit formulas for the dimension(s) (see Theorems 1,3 and 5). We also provide conditions for strict positivity and boundedness of the Hausdorff measure of the limit set (see Theorems 2 and 5). 
One very particular case is when a geometric construction is effected by a finite collection of similarity maps (affine contractions) $h_{1}, \ldots, h_{p}$ such that

$$
\Delta_{i_{1} \cdots i_{n}}=h_{i_{1}} \circ \cdots \circ h_{i_{n}}(\Delta),
$$

where $\Delta$ denotes a ball in $\mathbb{R}^{n}$ (see Sect. 4 ). Such a construction is called a similarity construction since its limit set $F$ is self similar, i.e., $F=\bigcup_{i=1}^{p} h_{i}(F)$. More generally, a geometric construction can be generated by a finite collection of contraction maps. These special constructions are particular cases of Moran geometric constructions. They have been the main objects of study in dimension theory for many years.

We will use two methods to obtain a lower bound for the Hausdorff dimension of a set: the uniform and non-uniform mass distribution principles. See Appendix 2. The uniform mass distribution principle requires the existence of a measure $m$ for which

$$
m(B(x, r)) \leqq C r^{s},
$$

where $B(x, r)$ is the ball of radius $r$ centered at the point $x \in F$ and $C$ is a constant. Then $s$ produces a lower bound for $\operatorname{dim}_{H} F$, the Hausdorff dimension of $F$. We stress that (2) must hold for all $x \in F$ and the constant $C$ must be independent of $x$ and $r$. The non-uniform mass distribution principle requires that (2) hold for $m$-almost every $x \in F$ with $C=C(x)$ a measurable function. Again, $s$ provides a lower bound for the Hausdorff dimension of the limit set. If the uniform mass distribution principle holds, one can obtain refined information about the $s$-Hausdorff measure of $F$. Although uniform mass distribution is stronger than the non-uniform version, we will establish it for a broad class of geometric constructions.

One can not expect to obtain any refined estimates for the Hausdorff and box dimensions of the limit set $F$ of a construction with arbitrary shape and spacing of the basic sets. We control the geometry of the construction by either restricting the shapes or sizes of the basic sets, the spacing of the basics sets, or both. If one has strong control over the sizes of the basic sets, then the spacing can be fairly arbitrary, and vice-versa. In this paper we introduce a new approach to studying geometric constructions having complicated geometry. Our approach is based on the notions of regularity and boundedness of the construction. Regular and bounded constructions are those which admit Moran covers, i.e., where the control over the geometry is effected in the spirit of the Moran approach by generalized ratio coefficients that encode the information about both the shape and spacing of the basic sets (Example 3 in Sect. 5 illustrates the role of spacing). For some constructions these coefficients are determined by the largest inscribed balls and smallest circumscribed balls for the basic sets. However, we construct an example where these numbers are completely independent of these balls (see Sect. 5, Example 8). We will compute the generalized ratio coefficients for all previously studied classes of geometric constructions as well as several new ones.

In [Ba], Barreira carried out an extensive analysis of the notion of regularity and formulated a general criterion for a geometric construction to be regular. $\mathrm{He}$ also constructed examples which illustrate that even a slight weakening of our regularity condition may cause a failure of our results.

One new class of geometric constructions that we introduce is asymptotic geometric constructions where the ratio coefficients depend on steps of the construction and admit a good asymptotic behavior. We apply our techniques to study asymptotic constructions and show that the non-uniform mass distribution principle can be 
used to estimate the Hausdorff dimension of the limit set. For asymptotic constructions, one can not expect the uniform mass distribution principle to hold. Hence the Hausdorff measure is usually zero or infinite, even in simple examples (see Sect. 5, Example 4). In order to obtain more information about the Hausdorff measure one can use a gauge function (see Appendix 4). In Sect. 5, we present a family of simple asymptotic geometric constructions which admit infinitely many gauge functions depending on the rate of convergence of ratio coefficients. This illustrates that the asymptotic category of constructions is quite rich in that there exists a large variety of different limit sets exhibiting many different structural properties.

We apply our study of asymptotic constructions to analyze random Moran geometric constructions. These constructions are essentially Moran construction with ratio coefficients chosen randomly from an arbitrary ergodic stationary process. We also consider random one-dimensional constructions. In the literature, random constructions are usually considered as a distinct class of geometric constructions. We stress that our approach completely unifies the analysis of many disparate types of geometric constructions.

For the Moran-like geometric constructions which we introduce, the crucial observation is the existence of a measure $m$ on the set $F$ such that the Hausdorff dimension of $F$ is equal to the Hausdorff dimension of $m$. This measure is the push forward of the equilibrium measure $\mu$ for the function $\phi(\omega)=s \log \lambda_{i_{1}}$, where $x$ is associated with a sequence $\omega=\left(i_{1} i_{2} \cdots\right)$ and $s$ is the unique root of the equation

$$
P(s \phi)=0,
$$

where $P$ is the thermodynamic pressure (see Appendix 3). For the Moran constructions, $s$ is the Hausdorff dimension of the limit set as well as its box dimension. We show that this is true for general symbolic Moran-like constructions (see Proposition 3 and Theorem 5).

Equation (3) was discovered by Bowen [Bo2] and seems to be universal: all known equations previously used to compute the Hausdorff dimension (for example Eq. (1)) coincide with or are particular cases of (3).

For the general symbolic geometric constructions the measure $\mu$ is an equilibrium measure and $m$ admits the non-uniform mass distribution principle. There is a crucial difference between Gibbs measures and equilibrium measures in Statistical Physics (see [R] and Appendix 3; see also Sect. 1, Remark 1). These notions coincide for subshifts of finite type, but need not coincide for general symbolic systems. We show that if $\mu$ is a Gibbs measure, then the Hausdorff dimension of the limit set, $s$, can be studied using the uniform mass distribution principle, and the $s$-dimensional Hausdorff measure is positive and finite (see Theorems 2 and 4). If $\mu$ is an equilibrium measure, then a non-uniform mass distribution principle can be applied to the measure $m$ (see Theorem 1).

Theorem 5 establishes the coincidence of the Hausdorff dimension and the box dimension of a measure and is similar in spirit to some results of Ledrappier and Young [LY]. It supports a general belief that the coincidence of the Hausdorff dimension and box dimension of a set is a rare phenomenon and requires rigid geometric constructions like some of those considered in this paper. In Sect. 5, we provide an example of a simple geometric construction in the plane with basic sets being rectangles for which the Hausdorff dimension $\operatorname{dim}_{H} F$, the lower box dimension $\operatorname{dim}_{B} F$, and the upper box dimension $\overline{\operatorname{dim}}_{B} F$, are distinct. The construction uses different ratio coefficients in different directions and combines both vertical and 
horizontal stacking of rectangles to effect the noncoincidence of the dimensions. In [PoW], the authors found that number theoretic properties of the ratio coefficients, related to Pisot numbers, is another mechanism to cause noncoincidence of dimensions. Another example which illustrates the non-coincidence of the Hausdorff and box dimensions was constructed in [Mc].

The coincidence of the Hausdorff dimension and box dimension of a measure is more common. There is a general criterion proved by Young [Y] that guarantees the coincidence. Namely, let $m$ be a Borel measure on $F$ such that for $m$-almost every $x \in F$ the limit

$$
\lim _{r \rightarrow 0} \frac{\log m(B(x, r))}{\log r}=d_{m}(x)
$$

exists. The limit $d_{m}(x)$ is called the pointwise dimension at $x$. In this case $m$ is called exact dimensional. If the limit does not exist, one can consider the lower and upper limits $\underline{d}_{m}(x), \bar{d}_{m}(x)$ to obtain the lower and upper pointwise dimensions at $x$. If for $m$-almost every $x$

$$
d_{m}(x)=\mathrm{const} \stackrel{\text { def }}{\equiv} s
$$

then the Hausdorff dimension of $m, \operatorname{dim}_{H} m$, and the lower and upper box dimensions of $m, \operatorname{dim}_{B} m$ and $\overline{\operatorname{dim}}_{B} m$, coincide and have the common value $s$.

This statement poses the problem of whether a given measure $m$ is exact dimensional and moreover whether $d_{m}(x)=$ const almost everywhere.

To give some insight into this problem consider a measure supported on the limit set of a Moran-like geometric construction. It is not difficult to show that the limit

$$
\lim _{n \rightarrow \infty} \frac{\log m\left(\Delta_{n}(x)\right)}{\log \operatorname{diam}\left(\Delta_{n}(x)\right)}
$$

exists almost everywhere, where $\Delta_{n}(x)$ is the basic set at step $n$ containing $x$. In Sect. 6 we develop a new technique to show that for any measure supported on the limit set of a Moran-like geometric construction, the above symbolic pointwise dimension coincides with the pointwise dimension of $m$ (see Sect. 6.4). This result has only been known in a simple particular case when the construction is effected by similarity maps.

For measures invariant under dynamical systems the problem is more subtle. In [C], Cutler constructed an example of a continuous map that preserves an ergodic exact dimensional measure $m$ with $d_{m}(x)$ essentially non-constant. In Sect. 6.4, we present a much simpler version of the construction of such a map that uses a special simple geometric construction. In addition, we show that such a map can be arranged to be Hölder continuous and topologically hyperbolic (i.e. satisfying the Axiom $\mathrm{A}^{\#}$ property, see $[\mathrm{AJ}]$ ).

If the map is smooth and $m$ is exact dimensional and ergodic, then $d_{m}(x)=$ const almost everywhere (since $d_{m}(x)$ is invariant under the map and measurable). Ledrappier and Misiurewicz [LM] constructed a one-dimensional smooth map preserving an ergodic measure that is not exact dimensional. Eckmann and Ruelle conjectured that an ergodic measure invariant under a $C^{2}$-diffeomorphism with non-zero Lyapunov exponents is exact dimensional (and, hence, $d_{m}(x)=$ const, see [ER]). This is now one of the most challenging, still open problems in the interface between dimension theory and dynamical systems. In [Y], Young obtained the positive solution for the Eckmann-Ruelle conjecture in the two-dimensional case. In [L], Ledrappier 
proved the conjecture for Bowen-Ruelle-Sinai measures and in [PY], Pesin and Yue extended this result to some measures including Gibbs measures for Axiom A diffeomorphisms.

In Sect. 6, we obtain the positive solution of the conjecture for Gibbs measures for conformal Hölder continuous expanding maps and conformal Axiom $\mathrm{A}^{\#}$ homeomorphisms. On the other hand, we construct a Hölder continuous topologically hyperbolic homeomorphism of positive topological entropy for which the unique measure of maximal entropy is ergodic and has different upper and lower pointwise dimensions almost everywhere (see Theorem 9). In other words, the Hölder continuous version of the Eckmann-Ruelle Conjecture fails.

Edgar and Mauldin [EM] computed the Hausdorff dimension for Markov constructions generated by a finite collection of similarity maps. Stella [St] obtained estimates for the Hausdorff and box dimensions of limit sets for Moran-like constructions with non-overlapping basic sets modeled by subshifts of finite type, which is our Corollary 1. Afraimovich and Shereshevsky [AS] found a lower estimate for the Hausdorff dimension of some simple geometric constructions. Similar types of simple geometric constructions, given by two-dimensional self-affine maps related to graphs of functions, were considered by Bedford and Urbanski in [BU]. Shereshevsky [S] also considered some one-dimensional Markov geometric constructions. We are aware of no author who considered any type of geometric construction based on a symbolic system more general than a subshift of finite type.

Various geometric constructions with randomly chosen ratio coefficients have been considered by Falconer [F2], Graf [G], Kahane [K], Graf, Mauldin and Williams [GMW], and Mauldin and Williams [MW2]. These authors studied random geometric constructions whose basic sets are geometrically similar. These constructions are modeled by branching processes that correspond to the full shift on $p$ symbols with $p^{n}$ ratio coefficients at step $n$ chosen randomly, essentially independently and with the same distribution on $(0,1)$. They also assume independence conditions over $n$. In this paper, we consider branching processes that are associated with arbitrary compact shift-invariant subsets. We generate the ratio coefficients by choosing $p$ random numbers on the interval $(a, b)$ where $0<a \leqq b<1$. We do not require that ratio coefficients be independent nor be identically distributed.

\section{Geometric Constructions}

We define geometric constructions of Cantor-like subsets of $\mathbb{R}^{d}$ by using a symbolic description in the space of all one-sided infinite sequences $\left(i_{1} i_{2} \cdots\right)$ on $p$ symbols. We denote this space by $\Sigma_{p}^{+}$and endow it with its usual topology (see Appendix 3 ).

A symbolic construction is defined by

a) a compact set $Q \subset \Sigma_{p}^{+}$invariant under the shift $\sigma$ (i.e., $\sigma(Q)=Q$ ) such that $\sigma \mid Q$ is topologically transitive

b) a family of compact sets called basic sets $\left\{\Delta_{i_{1} \cdots i_{n}}\right\} \subset \mathbb{R}^{d}$ for $i_{j}=1,2, \ldots, p$ and $n \in \mathbb{N}$ where the $n$-tuples $\left(i_{1} \cdots i_{n}\right)$ are admissible with respect to $Q$ (i.e., there exists $\omega=\left(i_{1}^{\prime}, i_{2}^{\prime}, \cdots\right) \in Q$ such that $\left.i_{1}^{\prime}=i_{1}, i_{2}^{\prime}=i_{2}, \ldots, i_{n}^{\prime}=i_{n}\right)$ and these sets satisfy

$$
\lim _{n \rightarrow \infty} \max _{\left(i_{1} \cdots i_{n}\right)} \operatorname{diam}\left(\Delta_{i_{1} \cdots i_{n}}\right)=0 .
$$


For any admissible sequence $\left(i_{1} \cdots i_{n+1}\right) \in\{1, \ldots, p\}^{n}$, we require that

$$
\Delta_{i_{1} \cdots i_{n} i_{n+1}} \subset \Delta_{i_{1} \cdots i_{n}}
$$

The limit set $F$ for this construction is defined by

$$
F=\bigcap_{n=1}^{\infty} \bigcup_{\substack{\left(t_{1} \\\right. \text { admissible }}} \Delta_{i_{1} \cdots i_{n}} .
$$

The limit set $F$ is compact. We stress that we make no assumption on the topology and geometry of the basic sets $\left\{\Delta_{i_{1} \cdots i_{n}}\right\}$ other than they are compact subsets of Euclidean space. In particular, they need not be connected and their boundaries may be fractal. We also stress that the placement of the basic sets is completely arbitrary and basic sets on the same level may intersect.

There is a particular but important case when the basic sets on the same level are disjoint, i.e.,

$$
\Delta_{i_{1} \cdots i_{n}} \cap \Delta_{i_{1}^{\prime} \cdots i_{n}^{\prime}}=\emptyset \quad \text { if }\left(i_{1} \cdots i_{n}\right) \neq\left(i_{1}^{\prime}, \ldots, i_{n}^{\prime}\right) .
$$

Condition (5) is known as the strong separation condition. If it holds then $F$ is a generalized Cantor set, i.e., it is a perfect, nowhere dense, and totally disconnected set. We also consider a weaker version of condition 5 known as the separation condition where

$$
\Delta_{i_{1} \cdots i_{n}} \cap \Delta_{i_{1}^{\prime} \cdots i_{n}^{\prime}} \cap F=\emptyset \quad \text { if }\left(i_{1} \cdots i_{n}\right) \neq\left(i_{1}^{\prime} \cdots i_{n}^{\prime}\right) .
$$

We define a coding map $\chi: Q \rightarrow F$ by associating to a given point $\omega=$ $\left(i_{1} i_{2} \cdots\right) \in Q$, the point $x=\bigcap_{k=1}^{\infty} \Delta_{i_{1} \cdots i_{k}}$. This map is a continuous surjection.

Consider the symbolic dynamical system $(Q, \sigma)$, where $Q \subset \Sigma_{p}^{+}$. Given a $p$-tuple $\alpha=\left(\alpha_{1}, \ldots, \alpha_{p}\right)$ such that $0<\alpha_{i}<1$, there exists a uniquely defined number $s_{\alpha}$ such that $P\left(s_{\alpha} \log \alpha_{i_{1}}\right)=0$, where $P$ denotes the topological pressure (see Appendix 3). Let $\mu_{\alpha}$ denote an equilibrium measure for the function $\left(i_{1}, i_{2}, \ldots\right) \mapsto$ $s_{\alpha} \log \alpha_{i_{1}}$ on $Q$, and let $m_{\alpha}$ be the push forward measure on $F$ under the coding map $\chi$.

1. Lower Estimates for the Hausdorff Dimension. Since we allow arbitrary spacing and shape of the basic sets, one can not expect, in general, to obtain any refined estimates for the Hausdorff and box dimensions of the limit set $F$ (see Sect. 5, Example 8). To obtain refined estimates, one needs to control the geometry of the construction by either controlling the spacing, sizes or shapes of the basic sets. If one has strong control over the sizes of the basic sets, then the spacing can be fairly arbitrary, and vice-versa. One well known method to control the geometry is to effect the construction using similarity maps (see Sect. 4). This class of construction is very restrictive since one has strong control over both the spacing of the basic sets and their sizes in all directions.

We will present a general method to control the geometry of the construction and extend the Moran approach from the full shift to a general symbolic system. This requires the non-uniform mass distribution principle (instead of the uniform one). We will estimate the dimensions for a large class of constructions called regular constructions which include many constructions where one has only moderate control over the spacing and/or the sizes of the basic sets. 
In a regular construction, control over the geometry is given by numbers $\gamma_{1}, \ldots, \gamma_{p}$ such that one can approximate the basic sets $\Delta_{i_{1} \cdots i_{n}}$ by balls of radius $\prod_{j=1}^{n} \gamma_{i}$. We believe that one can compute the numbers $\gamma_{1}, \ldots, \gamma_{p}$ and construct the balls using detailed information about shape and spacing of the basic sets. In some cases these balls coincide with the largest balls that can be inscribed in the basic sets. However, Example 8 of Sect. 5 illustrates that the optimal numbers $\gamma_{1}, \ldots, \gamma_{p}$ may be completely independent of the radius of the largest inscribed balls. We will compute the numbers $\gamma_{1}, \ldots, \gamma_{p}$ for all previously studied classes of geometric constructions as well as several new ones, and we will produce refined estimates for the Hausdorff dimension from below.

Let $Z \subset Q$. Given $0<r<1$ and a vector of numbers $\gamma=\left(\gamma_{1}, \ldots, \gamma_{p}\right)$, $0<\gamma_{i}<1, i=1, \ldots, p$, we first define a special cover $\mathfrak{U}_{r, Z}=\mathfrak{U}_{r, Z}(\gamma)$ of the set $Z$. For any $\omega \in Z$, let $n(\omega)$ denote the unique positive integer such that $\gamma_{i_{1}} \gamma_{i_{2}} \cdots \gamma_{i_{n(\omega)}}>r$ and $\gamma_{i_{1}} \gamma_{i_{2}} \cdots \gamma_{i_{n(\omega)+1}} \leqq r$. It is easy to see that $n(\omega) \rightarrow \infty$ as $r \rightarrow 0$ uniformly in $\omega$. Fix $\omega \in Z$ and consider the cylinder set $C_{i_{1} \cdots l_{n(\omega)}}$. We have $\omega \in C_{i_{1} \cdots i_{n(\omega)}}$, and if $\omega^{\prime} \in C_{i_{1} \cdots i_{n(\omega)}} \cap Z$ and $n\left(\omega^{\prime}\right) \geqq n(\omega)$, then

$$
C_{i_{1} \cdots i_{n\left(\omega^{\prime}\right)}} \cap Z \subset C_{i_{1} \cdots i_{n(\omega)}} \cap Z
$$

Let $C(\omega)$ be the largest cylinder set containing $\omega$ with the property that $C(\omega)=$ $C_{i_{1} \cdots i_{n\left(\omega^{\prime \prime}\right)}}$ for some $\omega^{\prime \prime} \in C(\omega)$ and $C_{i_{1} \cdots i_{n\left(\omega^{\prime}\right)}} \cap Z \subset C(\omega) \cap Z$ for any $\omega^{\prime} \in C(\omega) \cap Z$. The sets $C(\omega) \cap Z$ corresponding to different $\omega \in Z$ either coincide or are disjoint. We call these sets $C_{r}^{(j)}, j=1, \ldots, N_{r}$. There exist points $\omega_{j} \in Z$ such that $C_{r}^{(j)}=C_{i_{1} \cdots i_{n\left(\omega_{j}\right)}}$. These sets form a cover of $Z$. Let $\Delta_{r}^{(j)}=\chi\left(C_{r}^{(j)}\right)$. These sets form a cover of the set $\chi(Z)$ which we call the Moran cover. In the case that $Z=Q$ we denote the above cover by $\mathfrak{U}_{r}=\mathfrak{U}_{r}(\gamma)$.

Consider the open Euclidean ball $B(x, r)$ of radius $r$ centered at a point $x$. Let $N(x, r)$ denote the number of sets $\Delta_{r}^{(j)}$ that have non-empty intersection with $B(x, r)$. We call a vector, $\gamma$, l-estimating if $N(x, r) \leqq M$, where $M$ is a constant independent of $x$ and $r$. We call this constant $M$ the Moran multiplicity factor. We call a symbolic construction regular if it admits an l-estimating vector. If $\gamma=\left(\gamma_{1}, \ldots, \gamma_{p}\right)$ is an l-estimating vector for a regular symbolic construction, then any vector $\tilde{\gamma}=$ $\left(\tilde{\gamma}_{1}, \ldots, \tilde{\gamma}_{p}\right)$ for which $\gamma_{i} \geqq \tilde{\gamma}_{i}, i=1, \ldots, p$ is also l-estimating. It is easy to see that $s_{\gamma} \leqq s_{\gamma}$. On the other hand a vector $\tilde{\gamma}=\left(\tilde{\gamma}_{1}, \ldots, \tilde{\gamma}_{p}\right)$ for which $\tilde{\gamma}_{i}$ is sufficiently close to 1 for some $i$ is not l-estimating.

Below we will provide several examples of regular constructions although there are many constructions which are not regular. For example, if the Hausdorff dimension of the limit set is zero, then the corresponding construction is not regular. Let $F_{1}, F_{2}$ be two limit sets for two symbolic constructions on the line: the first construction is defined on the interval $[0,1]$ and the second one on the interval $[2,3]$. It is easy to see that if $\operatorname{dim}_{H} F_{1}=0$ and $\operatorname{dim}_{H} F_{2}>0$ then the set $F_{1} \cup F_{2}$ is the limit set for a construction which is not regular but whose limit set has positive Hausdorff dimension.

In Sect. 3 we study the regularity of a large class of one-dimensional geometric constructions. One can see that a one dimensional geometric construction is regular if the basic sets are disjoint (see (5)) and satisfy

$$
\sup _{n} \sup _{\left(l_{1} l_{2} \cdots l_{n}\right)} \frac{\log \operatorname{diam}\left(\Delta_{i_{1} i_{2} \cdots i_{n}}\right)}{-n} \leqq \text { constant . }
$$


This condition provides strong control over the ratio coefficients and requires them to be uniformly bounded away from zero. It is easy to show that if the ratio coefficients go to zero uniformly in $n$, then the Hausdorff dimension of the limit set is zero.

Barreira [Ba] has shown that the above assumption is almost optimal. He exhibited a one dimensional simple geometric construction with ratio coefficients not uniformly bounded away from zero such that: (1) the construction is not regular, (2) the limit set $F$ satisfies the following strong homogeneity property:

$$
\operatorname{dim}_{H}(F \cap B(x, r))=\text { constant }>0
$$

for all $x \in F$ and $r>0$.

If one considers a geometric construction in the plane with limit set $F=F_{1} \times F_{2}$, where $F_{1}$ and $F_{2}$ are limit sets for one-dimensional geometric constructions and $\operatorname{dim}_{H} F_{1}=0$, then the construction is not regular. We believe that a geometric construction is regular if the projection of the basic sets at step $n$ onto every line satisfies the above uniformity condition uniformly over the direction of the projection.

Although the coding map for a regular symbolic geometric construction need not be injective, one can verify that the cardinality of each fibre $\left(\chi^{-1}(x), x \in F\right)$ is uniformly bounded by the Moran multiplicity factor $M$.

Theorem 1. Let $F$ be the limit set for a regular symbolic construction. Then $s_{\gamma} \leqq \operatorname{dim}_{H} F$ for any l-estimating vector $\gamma$. Hence, $\sup s_{\gamma} \leqq \operatorname{dim}_{H} F$, where the supremum is taken over all l-estimating vectors $\gamma$. Moreover, there exists a probability measure $\xi$ such that $\sup s_{\gamma} \leqq \operatorname{dim}_{H} \xi$, where $\operatorname{dim}_{H} \xi$ is defined as $\inf _{U}\left\{\operatorname{dim}_{H} U, \xi(U)=1\right\}$.

We stress that we did not require the strong separation condition (5). A more delicate question in dimension theory is whether the Hausdorff measure of the limit set is positive. The answer is presumably negative for a general symbolic geometric construction. For a regular construction, the positivity of the Hausdorff measure may depend on whether the equilibrium measure $\mu_{\gamma}$ is a Gibbs measure. If this measure is Gibbs, then $m_{\gamma}$ satisfies the uniform mass distribution principle and we have our most refined estimates. If the construction is modeled by a subshift of finite type (i.e., $Q=\Sigma_{A}^{+}$, where $A$ is an irreducible transfer matrix) or a sophic system (a finite factor of a subshift of finite type), then the measure $\mu_{\gamma}$ is Gibbs. We denote by $m_{H}(t, F)$ the $t$-dimensional Hausdorff measure of $F$ (see Appendix 1).

Theorem 2. Let $F$ be the limit set of a regular symbolic construction. Assume that there exists an l-estimating vector $\gamma$ such that the measure $\mu_{\gamma}$ on $Q$ is a Gibbs measure. Then

1) the measure $m_{\gamma}$ satisfies the uniform mass distribution principle,

2) $0<m_{H}\left(s_{\gamma}, F\right)$, moreover, $m_{\gamma}(Z) \leqq C m_{H}\left(s_{\gamma}, Z\right)$ for any $Z \subset F$ where $C>0$ is a constant,

3) $s_{\gamma} \leqq \underline{d}_{m_{\gamma}}(x)$ for every $x \in F$.

The second statement in Theorem 2 is nontrivial only when $s_{\gamma}=\operatorname{dim}_{H} F$. Otherwise, $m_{H}\left(s_{\gamma}, F\right)=\infty$. If $s_{\gamma}<s=\operatorname{dim}_{H} F$, then the $s$-Hausdorff measure may be zero or infinite.

2. Upper Estimates for the upper box Dimension. In order to obtain upper estimates for the upper box dimension, we require that the diameters of the basic sets decrease exponentially. 
More precisely, we say that a vector $\lambda=\left(\lambda_{i}, \ldots, \lambda_{p}\right), 0<\lambda_{i}<1$ is a u-estimating vector for a construction if

$$
\operatorname{diam}\left(\Delta_{i_{1} \cdots i_{n}}\right) \leqq C \prod_{j=1}^{n} \lambda_{i_{j}},
$$

where $C>0$ is a constant. The symbolic construction is called bounded if there exists a $\mathrm{u}$-estimating vector $\lambda$. It is easy to see that if $\lambda=\left(\lambda_{1}, \ldots, \lambda_{p}\right)$ is a $\mathrm{u}$ estimating vector for a bounded construction, then any vector $\tilde{\lambda}_{2}\left(\tilde{\lambda}_{1}, \ldots, \tilde{\lambda}_{p}\right)$ for which $\tilde{\lambda}_{i} \geqq \lambda_{i}, i=1, \ldots, p$ is a u-estimating vector and $s_{\lambda} \leqq s_{\tilde{\lambda}}$.

We now wish to estimate the upper box dimension of the limit set $F$ for a symbolic geometric construction. We need not assume that the construction is regular, but only bounded.

Theorem 3. Let $F$ be the limit set for a bounded symbolic construction. Then

(1) $\overline{\operatorname{dim}}_{B} F \leqq s_{\lambda}$ for any u-estimating vector $\lambda$. Hence, $\overline{\operatorname{dim}}_{B} F \leqq \inf s_{\lambda}$, where the infinum is taken over all u-estimating vectors $\lambda$,

(2) $\bar{d}_{m_{i}}(x) \leqq s_{\lambda}$ for any $u$-estimating vector $\lambda$ and $m_{\lambda}$-almost every $x \in F$.

In the case when the measure $\mu_{\lambda}$ is a Gibbs measure, we can establish finiteness of the $s_{\lambda}$-Hausdorff measure and obtain a stronger statement about the upper pointwise dimensions.

Theorem 4. Let $F$ be the limit set for a bounded symbolic construction. Assume that there exists a u-estimating vector $\lambda$ such that the measure $\mu_{\lambda}$ on $Q$ is a Gibbs measure. Then

(1) $m_{H}\left(s_{\lambda}, F\right)<\infty$, moreover $m_{H}\left(s_{\lambda}, Z\right) \leqq C m_{\lambda}(Z)$ for any $Z \subset F$ where $C>0$ is a constant,

(2) $\bar{d}_{m},(x) \leqq s_{\lambda}$ for every $x \in F$.

The next statement provides an upper estimate for the number $s_{\lambda}$.

Proposition 1. Let $F$ be the limit set for a bounded symbolic construction and $\lambda$ a u-estimating vector.

(1) We have

$$
s_{\lambda} \leqq \frac{h(\sigma \mid Q)}{-\log \lambda_{\max }},
$$

where $\lambda_{\max }=\max _{k=1}^{p}\left\{\lambda_{k}\right\}$, and $h(\sigma \mid Q)$ denotes the topological entropy. Equality occurs if $\lambda_{i}=\lambda$ for $i=1, \ldots, p$.

(2) See [Fu]. If $\lambda_{i}=\lambda$ for $i=1, \ldots, p$, then

$$
s_{\lambda}=\operatorname{dim}_{H} F=\underline{\operatorname{dim}}_{B} F=\overline{\operatorname{dim}}_{B} F=\frac{h(\sigma \mid Q)}{-\log \lambda} .
$$

(3) If $h(\sigma \mid Q)=0$, then $s_{\lambda}=\operatorname{dim}_{H} F=\underline{\operatorname{dim}}_{B} F=\overline{\operatorname{dim}}_{B} F=0$.

The following theorem is an immediate consequence of Theorems 1-4. 
Theorem 5. Let $F$ be the limit set for a regular and bounded symbolic construction. Then for any l-estimating vector $\gamma$ and any u-estimating vector $\lambda$ we have

(1) $s_{i,} \leqq \operatorname{dim}_{H} F \leqq \operatorname{dim}_{B} F \leqq \overline{\operatorname{dim}}_{B} F \leqq s_{j}$, where $s_{; ;}$and $s_{j}$ are the unique roots of the equations $P\left(s_{i} \log \gamma_{i_{1}}\right)=0$ and $P\left(s_{i} \log \lambda_{l_{1}}\right)=0$, respectively,

(2) if $\gamma_{1}=\lambda_{1}$ for $i=1, \ldots, p$ then

$$
s=s_{\gamma^{\prime}}=s_{i}=\operatorname{dim}_{H} F=\underline{\operatorname{dim}}_{B} F=\overline{\operatorname{dim}}_{B} F .
$$

Moreover, $m=m_{i}=m_{i}$, and if the measure $\mu_{i}=\mu_{\gamma}$ is Gibbs, then $m_{H}(s, *)$ is equivalent to $m$ and $\underline{d}_{m}(x)=\bar{d}_{m}(x)=s$ for every $x \in F$.

In general, the lower and upper pointwise dimensions $\underline{d}_{m .}(x)$ and $\bar{d}_{m_{i}}(x)$ (as well as $\underline{d}_{m}(x)$ and $\left.\bar{d}_{m},(x)\right)$ do not coincide almost everywhere (see Sect. 6.2 and 6.3).

3. Markov construction. We will consider two important special cases of symbolic constructions specified by a subshift of finite type or the full shift. Let $A$ denote a $p \times p$ transfer matrix with entries $A(i, j)=0$ or 1 and let $\Sigma_{A}^{+}$consist of admissible sequences $\left(i_{1} i_{2} \cdots\right)$ with respect to $A$ (i.e., $A\left(i_{j}, i_{j+1}\right)=1$ for $\left.j=1,2 \cdots\right)$. The construction is called Markov if $Q=\Sigma_{A}^{+}$. In the case when the set $Q=\Sigma_{p}^{+}$the construction is called simple.

Consider a subshift of finite type $\left(\Sigma_{A}^{+}, \sigma\right)$. Given $p$ numbers $0<\alpha_{1}, \ldots, \alpha_{p}<1$, we define a $(p \times p)$ diagonal matrix $M_{t}(\alpha)=\operatorname{diag}\left(\alpha_{1}^{t}, \ldots, \alpha_{p}^{t}\right)$. Let $\rho(B)$ denote the spectral radius of the matrix $B$.

Proposition 2. The equation $P\left(s \log \alpha_{l_{1}}\right)=0$ is equivalent to the equation $\rho\left(A^{*} M_{s}(\alpha)\right)=1$, where $A^{*}$ denotes the transpose of the matrix $A$.

We will assume that the transfer matrix $A$ is irreducible. Then the shift map $\sigma: \Sigma_{A}^{+} \rightarrow \Sigma_{A}^{+}$is topologically transitive.

The following corollaries are immediate consequences of Theorem 5 and Proposition 2.

Corollary 1. Let $F$ be the limit set for a Markov regular and bounded construction. Then for any l-estimating vector $\gamma$ and any u-estimating vector $i$ we have

$$
s_{i} \leqq \operatorname{dim}_{H} F \leqq \operatorname{dim}_{B} F \leqq \overline{\operatorname{dim}}_{B} F \leqq s_{;},
$$

where $s_{;}$and $s$; are the unique roots of the equations

$$
\rho\left(A^{*} M_{s}(\gamma)\right)=1, \quad \rho\left(A^{*} M_{s}(\lambda)\right)=1
$$

respectively.

Corollary 2. Let $F$ be the limit set for a simple regular and bounded construction. Then for any l-estimating vector $\gamma$ and any $u$-estimating vector $\lambda$ we have

(1) $s_{\gamma} \leqq \operatorname{dim}_{H} F \leqq \operatorname{dim}_{B} F \leqq \overline{\operatorname{dim}}_{B} F \leqq s_{;}$, where $s_{\gamma}$ and $s_{\gamma}$ are the unique roots of the equations

$$
\sum_{i=1}^{p} \gamma_{l}^{t}=1 \quad \text { and } \quad \sum_{i=1}^{p} \lambda_{l}^{t}=1 \text {, }
$$

respectively. 
(2) The Gibbs measures $\mu_{\gamma}$ and $\mu_{i}$ on $\Sigma_{p}^{+}$satisfy

$$
\mu_{\gamma}\left(C_{l_{1} \cdots i_{n}}\right)=\prod_{j=1}^{n} \gamma_{i_{j}}^{s_{*}} \quad \text { and } \quad \mu_{\lambda_{\lambda}}\left(C_{i_{1} \cdots i_{n}}\right)=\prod_{j=1}^{n} \lambda_{i_{j}}^{s_{\prime}} \text {, }
$$

where $C_{i_{1} \cdots i_{n}}$ is a cylinder set.

\section{Remarks.}

(1) It follows from the expansiveness of the shift map that for any general symbolic system and any continuous function, there exists an equilibrium measure corresponding to this function. However, in general, this measure need not be a Gibbs measure. It is well known that if a symbolic system has the specification property [KH], then any equilibrium measure is Gibbs and is unique provided the function is Hölder continuous [R]. Thus, Theorem 5 provides refined estimates for the Hausdorff and box dimensions as well as the Hausdorff measure for a symbolic geometric construction modeled by a symbolic system which satisfies the specification property.

Subshifts of finite type and their finite factors (sophic systems) are known to satisfy the specification property $[\mathrm{R}, \mathrm{We}]$. The only other examples we know are beta-shifts $[\mathrm{BM}]$ for special values of $\beta$.

If the topological entropy of $\sigma \mid Q$ is zero, then by Proposition 1, the Hausdorff dimension and box dimension coincide and are zero. In this case the measure $\mu_{;}$is a measure of maximal entropy and is not a Gibbs measure.

We believe that one can find a symbolic system with positive topological entropy and can build a $\gamma$-regular and $\lambda$-bounded symbolic construction such that the two measures $\mu_{\gamma}$ and $\mu_{i}$ are not Gibbs. Moreover, $\mu_{i}$ will not be equivalent to the $s_{i}$-Hausdorff measure. There is an interesting question of whether one can build a regular (or bounded) symbolic construction admitting two l-estimating vectors $\gamma_{1}, \gamma_{2}$ (two u-estimating vectors $\left.\lambda_{1}, \lambda_{2}\right)$ such that $\mu_{i_{1}}$ is Gibbs and $\mu_{\gamma_{2}}$ is not $\left(\mu_{i_{1}}\right.$ is a Gibbs measure and $\mu_{i_{2}}$ is not).

One can also obtain an appropriate version of Theorem 5 under this weaker hypothesis.

(2) In the Markov constructions above, we assumed that the $\operatorname{transfer~matrix~} A$ was transitive. For an arbitrary transfer matrix $A$, one can decompose the set $\Sigma_{A}^{+}$into two shift-invariant subsets: the wandering set $Q_{1}$ and the non-wandering set $Q_{2}$. The latter can be further partitioned into finitely many shift-invariant subsets of the form $\Sigma_{A_{i}}^{+}$, where each matrix $A_{i}$ is transitive and corresponds to a class of equivalent recurrent states $[\mathrm{AJ}]$. The limit set $F$ contains disjoint sets $F_{i}=\chi\left(\Sigma_{A_{l}}^{+}\right)$. Each set $F_{i}$ is the limit set for a Markov construction defined by the transitive matrix $A_{l}$ and hence admits lower and upper estimates for the Hausdorff and box dimensions stated in Corollary 1. In [MW1], the authors discuss the effect of the wandering set $Q_{1}$ on the dimension of the limit set $F$ in special cases.

(3) Consider the full shift $\sigma$ on $p$ symbols with the standard metric $d_{\beta}, \beta>1$. Let $\mu$ be a $\sigma$-invariant ergodic measure, and let $r=\beta^{-n}$. Since the ball centered at $\omega$ with radius $r, B(\omega, r)$, is a cylinder set, we have by a theorem of Brin and Katok [BK] that for $\mu$ almost every $\omega$,

$$
\frac{\log \mu(B(\omega, r))}{\log r}=-\frac{\log \mu\left(B\left(\omega, \beta^{-n}\right)\right)}{n \log \beta} \stackrel{n \rightarrow \infty}{\rightarrow} \frac{h_{\mu}(\sigma)}{\log \beta},
$$


hence

$$
\frac{h_{\mu}(\sigma)}{\log \beta}=\underline{d}_{\mu}(\omega)=\bar{d}_{\mu}(\omega)=\operatorname{dim}_{H} \mu=\underline{\operatorname{dim}}_{B} \mu=\overline{\operatorname{dim}}_{B} \mu,
$$

where $h_{\mu}(\sigma)$ denotes the Kolmogorov-Sinai entropy of the shift map. It is evident that the common value depends on $\beta$, which is not surprising since the two metrics $d_{\beta_{1}}$ and $d_{\beta_{2}}$ are not equivalent for $\beta_{1} \neq \beta_{2}$. Now consider the Moran simple construction with parameters $\lambda=\left(\lambda_{1}, \ldots, \lambda_{p}\right)$. Since $\mu_{\lambda}$ is Gibbs we have that $h_{\mu},(\sigma)+s \int \log \lambda_{\omega_{1}} d \mu_{\lambda}=0$, which gives

$$
s=-\frac{h_{\mu_{i}}(\sigma)}{\int \log \lambda_{i_{1}} d \mu_{\lambda}}=\frac{h_{\mu_{i}}(\sigma)}{\log \beta}
$$

if

$$
\beta=\exp \left(-\int \log \lambda_{i_{1}} d \mu_{\lambda}\right)
$$

Thus the Hausdorff dimension of $\mu_{\lambda}$ calculated with respect to the two metrics $d_{\beta}$ and $\chi^{*} \rho$ ( $\rho$ is the Euclidean metric on $\mathbb{R}^{d}$ and $\chi^{*} \rho\left(\omega_{1}, \omega_{2}\right)=\rho\left(\chi\left(\omega_{1}\right), \chi\left(\omega_{2}\right)\right.$ ) coincide. The two metrics $\chi^{*} \rho$ and $d_{\beta}$ with $\beta$ satisfying (6) are not equivalent if not all the $\lambda_{i}$ coincide. To see this, notice that $\chi: F \rightarrow \Sigma_{p}^{+}$is onto. We have with respect to the metric $\rho$,

$$
\operatorname{dim}_{H} F=s=\frac{h_{\mu_{i}}(\sigma)}{\log \beta},
$$

and with respect to the metric $d_{\beta}$

$$
\operatorname{dim}_{H} \Sigma_{p}^{+}=\frac{\log p}{\log \beta}=\frac{h(\sigma)}{\log \beta}
$$

where $h(\sigma)$ denotes the topological entropy of $\sigma$. It is easy to see that $h_{\mu_{i}}(\sigma)=h(\sigma)$ if and only if $\lambda_{1}=\lambda_{2}=\cdots=\lambda_{p}=\lambda$.

(4) Let $F$ be the limit set for a symbolic geometric construction and $m$ a measure on $F$. We define $K_{\alpha}=\left\{x \in F \mid d_{m}(x)=\underline{d}_{m}(x)=\bar{d}_{m}(x)=\alpha\right\}$ and $f(\alpha)=\operatorname{dim}_{H}\left(K_{\alpha}\right)$. We also define $H P_{m}(q)=\lim _{r \rightarrow 0} \frac{\log \int_{F} m\left(B(x, r)^{q} d m(x)\right.}{\log r}$, assuming that the limit exists [HP]. The function $f(\alpha)$ is known as the multifractal spectrum of $F$ and the function $H P_{m}(q)$ is known as the Hentschel-Procaccia spectrum for dimension $q$. These two functions play a prominent role in the description of the multifractal structure of the limit set. In some special cases, these functions form a Legendre transform pair [CLP, CM, Lo, OSY, PW2].

It follows from Statement 2 of Theorem 5 that if the construction is Moran and $\mu_{\lambda}$ is the Gibbs measure for the function $s \log \lambda_{i_{1}}$, then $f(\alpha)$ is a $\delta$-function $\left(f(\alpha)=s\right.$ if $\alpha=s$ and 0 otherwise) and $H P_{m_{i}}(q)=s q$. These functions form a degenerate Legendre transform pair. The most general results concerning the multifractal formalism for Moran symbolic geometric constructions (with arbitrary Gibbs measures) are obtained by the authors in [PW2]. 


\section{Examples of Symbolic Constructions}

Here we consider several classes of symbolic constructions with restrictions on the shapes of basic sets or restrictions on the gaps between basic sets.

1. Moran-like constructions. In this section we introduce a new class of symbolic geometric constructions with basic sets $\Delta_{i_{1} \cdots i_{n}}$ satisfying the following conditions:

$$
\underline{B}_{i_{1} \cdots i_{n}} \subset \Delta_{i_{1} \cdots i_{n}} \subset \bar{B}_{i_{1} \cdots i_{n}}
$$

where $\underline{B}_{i_{1} \cdots i_{n}}$ and $\bar{B}_{i_{1} \cdots i_{n}}$ are closed balls having radii $\underline{r}_{i_{1} \cdots i_{n}}$ and $\bar{r}_{i_{1} \cdots i_{n}}$ respectively,

(2) int $\underline{B}_{i_{1} \cdots i_{n}} \cap$ int $\underline{B}_{l_{1}^{\prime} \cdots i_{n}^{\prime}}=\emptyset$ if $\left(i_{1} \cdots i_{n}\right) \neq\left(i_{1}^{\prime}, \ldots, i_{n}^{\prime}\right)$, where int $R$ denotes the interior of the set $R$.

(3) $\underline{r}_{i_{1} \cdots i_{n}}=C_{1} \prod_{j=1}^{n} \lambda_{i_{j}}$ and $\bar{r}_{i_{1} \cdots i_{n}}=C_{2} \prod_{j=1}^{n} \lambda_{i_{j}}$, where $0<\lambda_{l}<1, i=1, \ldots, p$ and $C_{1}, C_{2}$ are positive constants.

This class of constructions includes Moran geometric constructions. Although basic sets of these constructions are essentially balls, their topology and geometry may be quite complicated (for example, they may not be connected and their boundary may be fractal). In particular the basic sets at level $n$ of the construction need not be geometrically similar to the basic sets at level $n-1$. Moreover, the basic sets at step $n$ of the construction may intersect.

Proposition 3. Let $F$ be the limit set for the construction defined above. Then

(1) the construction is regular and bounded with l-estimating vector and $u$-estimating vector equal to $\lambda=\left(\lambda_{1}, \ldots, \lambda_{p}\right)$,

(2) $s=s_{\lambda}=\operatorname{dim}_{H} F=\underline{\operatorname{dim}}_{B} F=\overline{\operatorname{dim}}_{B} F$. Moreover, if the measure $\mu_{\lambda}$ on $Q$ is Gibbs, then $m_{H}(s, *)$ is equivalent to $m_{\lambda}, 0<m_{H}(s, F)<\infty$, and $s=\underline{d}_{m_{i}}(x)=$ $\bar{d}_{m},(x)$ for every $x \in F$.

The first statement of Proposition 3 is obvious, and the second statement follows from Theorem 5 .

2. Constructions with rectangles. We now consider geometric constructions where the basic sets are (multi-dimensional) rectangles. More precisely, we call a symbolic construction a construction with rectangles if there exist $2 p$ numbers $\underline{\lambda}_{i}, \bar{\lambda}_{i}, i=$ $1, \ldots, p, 0<\underline{\lambda}_{i} \leqq \bar{\lambda}_{i}<1$ such that the basic set $\Delta_{i_{1} \cdots l_{n}} \subset \mathbb{R}^{d}$ is a rectangle (the direct product of intervals, called sides, lying on $n$ orthogonal lines) with the largest side equal to $C_{1} \prod_{j=1}^{n} \bar{\lambda}_{i}$, and the smallest side equal to $C_{2} \prod_{j=1}^{n} \underline{\lambda}_{i}$, where $C_{1}$ and $C_{2}$ are positive constants.

Proposition 4. Let $F$ be the limit set for a symbolic construction with rectangles satisfying the strong separation condition (5). Then

(1) the construction is regular and bounded with l-estimating vector $\underline{\lambda}=\left(\underline{\lambda}_{1}, \ldots\right.$, $\left.\underline{\lambda}_{p}\right)$ and $u$-estimating vector $\bar{\lambda}=\left(\bar{\lambda}_{1}, \ldots, \bar{\lambda}_{p}\right)$,

(2) $s_{\underline{\underline{\lambda}}} \leqq \operatorname{dim}_{H} F \leqq \underline{\operatorname{dim}}_{B} F \leqq \overline{\operatorname{dim}}_{B} F \leqq s_{\overline{\hat{\lambda}}}$. Moreover, if the measures $\mu_{\underline{\underline{\lambda}}}$ and $\mu_{\overline{\lambda_{j}}}$ on $Q$ are Gibbs, then $s_{\underline{\underline{\lambda}}} \leqq \underline{d}_{m_{-}}(x)$ and $\bar{d}_{m_{\bar{\lambda}}}(x) \leqq s_{\bar{\lambda}}$ for every $x \in F$. 
The first statment is obvious and the second statement follows from Theorems 1-4. In Sect. 5, Example 6, we will exhibit a simple construction with rectangles for which $\operatorname{dim}_{H} F<\underline{\operatorname{dim}}_{B} F<\overline{\operatorname{dim}}_{B} F$.

In [Mc], McMullen studied a special example of a geometric construction in the plane with rectangles. Given positive integers $m$ and $n, m \leqq n$, he considers the partition of the unit square into rectangles of size $\frac{1}{m} \times \frac{1}{n}$. One chooses any $r$, $1 \leqq r \leqq m \cdot n$ of these rectangles and colors them. One then linearly contracts the unit square by a factor of $\frac{1}{m} \times \frac{1}{n}$ and inserts a copy of this set into each of the original colored rectangles. One keeps going and obtains a limit set $F$. This is not a similarity construction since one does not insert a copy of this set into all of the original rectangles, just the shaded ones. McMullen proves that $\underline{\operatorname{dim}}_{B} F=$ $\overline{\operatorname{dim}}_{B} F \stackrel{\text { def }}{\equiv} \operatorname{dim}_{B} F$ and finds explicit formulas for the box dimension and the Hausdorff dimension which use information about the initial configuration. It follows from his results that for most initial configurations, $\operatorname{dim}_{H} F<\operatorname{dim}_{B} F$.

The vectors $\gamma=\left(\frac{1}{n}, \frac{1}{n}\right)$ and $\lambda=\left(\frac{1}{m}, \frac{1}{m}\right)$ are 1 -estimating and $\mathrm{u}$-estimating vectors respectively. Proposition 4 is applicable to this example and gives the following estimate:

$$
\frac{\log r}{-\log n} \leqq \operatorname{dim}_{H} F \leqq \operatorname{dim}_{B} F \leqq \frac{\log r}{-\log m}
$$

It follows from McMullen's formulas that for most initial configurations, each of the three inequalities may be strict.

In [PoW], the authors studied another type of simple geometric construction with rectangles in the plane. Their construction is the similarity construction with two rectangles, each of size $\lambda_{1} \times \lambda_{2}, \lambda_{1} \leqq \lambda_{2}$, whose boundaries are aligned with the coordinate axes. The vectors $\gamma=\left(\lambda_{1}, \lambda_{1}\right)$ and $\lambda=\left(\lambda_{2}, \lambda_{2}\right)$ are 1-estimating and $\mathrm{u}$-estimating vectors respectively. Proposition 4 is applicable to this example and gives the following estimate:

$$
\frac{\log 2}{-\log \lambda_{1}} \leqq \operatorname{dim}_{H} F \leqq \operatorname{dim}_{B} F \leqq \overline{\operatorname{dim}}_{B} F \leqq \frac{\log 2}{-\log \lambda_{2}}
$$

The authors proved that: 1) $\left.\underline{\operatorname{dim}}_{B} F=\overline{\operatorname{dim}}_{B} F \stackrel{\text { def }}{\equiv} \operatorname{dim}_{B} F, 2\right)$ for "almost all" initial configurations with $\lambda_{2} \leqq \frac{1}{2}, \operatorname{dim}_{H} F=\operatorname{dim}_{B} F=\frac{\log 2}{-\log \lambda_{2}}, 3$ ) for "almost all" initial configurations with $\lambda_{2}>\frac{1}{2}, \operatorname{dim}_{B} F=\frac{\log \left(\frac{2 i_{2}}{i_{1}}\right)}{-\log \lambda_{1}}$ and for a set of $\lambda_{2}$ of positive measure, $\left.\operatorname{dim}_{H} F=\operatorname{dim}_{B} F=\frac{\log \left(\frac{2 \lambda_{2}}{1}\right)}{-\log \lambda_{1}}, 4\right)$ if $\lambda_{2}$ is the reciprical of a Pisot number, then the Hausdorff dimension is strictly less than $\frac{\log \left(\frac{2 / 2}{\lambda_{1}}\right)}{-\log \lambda_{1}}$. Hence the Hausdorff dimension of the limit set may depend on delicate number theoretic properties of the ratio coefficients.

3. Constructions with exponentially large gaps. We now consider constructions where we have strong control over the gaps, but no control over the shape and the size of the basic sets. We call a symbolic construction a construction with exponentially large gaps if there exists a number $0<\beta<1$ such that the (Euclidean) distance between any two basic sets $\Delta_{i_{1} \cdots l_{n}}$ and $\Delta_{j_{1} \cdots j_{n}}$ is exponentially bounded away from 0 , i.e.,

$$
\operatorname{dist}\left(\Delta_{i_{1} \cdots i_{n}}, \Delta_{j_{1} \cdots j_{n}}\right) \geqq C \beta^{n},
$$

where $C>0$ is a constant. 
Proposition 5. Let $F$ be the limit set for a symbolic construction with exponentially large gaps. Then

(1) the construction is regular with l-estimating vector $\gamma=(\beta, \ldots, \beta)$.

(2) $s_{\gamma} \leqq \operatorname{dim}_{H} F$.

Statement 1 is obvious and the proof of Statement 2 follows immediately from Theorem 1.

4. One-dimensional constructions. We now consider one-dimensional symbolic constructions. We assume that each basic set $\Delta_{i_{1} \cdots i_{n}}$ is an interval satisfying

$$
\underline{I}_{i_{1} \cdots i_{n}} \subset \Delta_{i_{1} \cdots i_{n}} \subset \bar{I}_{i_{1} \cdots i_{n}},
$$

where $\underline{I}_{i_{1} \cdots i_{n}}$ and $\bar{I}_{i_{1} \cdots i_{n}}$ are closed intervals of length $\underline{l}_{i_{1} \cdots i_{n}}$ and $\bar{l}_{i_{1} \cdots l_{n}}$ respectively,

(2) int $\underline{I}_{i_{1} \cdots i_{n}} \cap \operatorname{int} \underline{I}_{i_{1}^{\prime} \cdots i_{n}^{\prime}}=\emptyset$ if $\left(i_{1} \cdots i_{n}\right) \neq\left(i_{1}^{\prime}, \ldots, i_{n}^{\prime}\right)$,

(3) $\underline{l}_{l_{1} \cdots l_{n}}=C_{1} \prod_{j=1}^{n} \underline{\lambda}_{l_{j}}$ and $\bar{l}_{i_{1} \cdots i_{n}}=C_{2} \prod_{j=1}^{n} \bar{\lambda}_{i_{j}}$, where $0<\underline{\lambda}_{l} \leqq \bar{\lambda}_{i}<1$, $i=1, \ldots, p$ and $C_{1}, C_{2}$ are positive constants.

Proposition 6. Let $F$ be the limit set for a one-dimensional symbolic construction defined above. Then

(1) the construction is regular with l-estimating vector $\underline{\lambda}=\left(\underline{\lambda}_{1}, \ldots, \underline{\lambda}_{p}\right)$ and bounded with u-estimating vector $\bar{\lambda}=\left(\bar{\lambda}_{1}, \ldots, \bar{\lambda}_{p}\right)$,

(2) $s_{\underline{\lambda}} \leqq \operatorname{dim}_{H} F \leqq \underline{\operatorname{dim}}_{B} F \leqq \overline{\operatorname{dim}}_{B} F \leqq s_{\bar{\lambda}}$,

(3) if $\underline{\lambda}_{l}=\bar{\lambda}_{i}=\lambda_{i}$, then $s=s_{\lambda}=\operatorname{dim}_{H} F=\underline{\operatorname{dim}}_{B} F=\overline{\operatorname{dim}}_{B} F$. Moreover, if the measure $\mu_{\lambda}$ on $Q$ is Gibbs, then $m_{H}(s, \cdot)$ is equivalent to $m_{\lambda}, 0<m_{H}(s, F)<\infty$, and $s=\underline{d}_{m},(x)=\bar{d}_{m},(x)$ for every $x \in F$.

The proof of Statement 1 is obvious and Statements 2 and 3 follow immediately from Theorems 1-5.

\section{Asymptotic and Random Symbolic Constructions}

3.1. We now consider more general types of geometric constructions where the basic sets are asymptotically balls. These constructions may not be regular, but are regular on each subset of an increasing sequence of subsets which exhaust the limit set up to a set of measure zero. More precisely we say that a vector $\gamma$ is a conditionally l-estimating vector for a symbolic construction if there exists a sequence of subsets $\left\{Q_{l}\right\}, l=1,2, \ldots$ such that

(1) $Q_{l} \subset Q_{l+1}$ and $\bigcup_{l} Q_{l}=Q$ up to a set of $\mu_{\eta}$-measure zero,

(2) there exist $l$ such that $\mu_{\gamma}\left(Q_{l}\right)>0$,

(3) for every $l=1,2, \ldots$ and $\varepsilon>0$ there exists $M(l, \varepsilon)$ such that for all $\omega \in Q_{l}$ and $r>0$,

$$
N(x, r) \leqq M(l, \varepsilon) r^{-\varepsilon},
$$


where $x=\chi(\omega)$ and $N(x, r)$ denotes the number of sets in the Moran cover of the set $Q_{l}$ (constructed in Sect. 1) that have nonempty intersection with $B(x, r) \cap \chi\left(Q_{l}\right)$.

We call a symbolic construction conditionally regular if it admits a conditionally 1-estimating vector.

One can generalize Theorem 1 to obtain the following result.

Theorem 6. Let $F$ be the limit set for a conditionally regular symbolic construction. Then $s_{\gamma} \leqq \operatorname{dim}_{H} F$, where $\gamma$ is a conditionally l-estimating vector.

We begin with an asymptotic version of symbolic constructions. We will show that these constructions are conditionally regular.

1. Moran-like asymptotic constructions. A Moran-like asymptotic symbolic construction is a symbolic construction with basic sets $\Delta_{i_{1} \cdots i_{n}}$ satisfying the following conditions:

(1)

$$
\underline{B}_{i_{1} \cdots i_{n}} \subset \Delta_{i_{1} \cdots i_{n}} \subset \bar{B}_{i_{1} \cdots i_{n}},
$$

where $\underline{B}_{i_{1} \cdots i_{n}}$ and $\bar{B}_{i_{1} \cdots i_{n}}$ are closed balls having radii $\underline{r}_{i_{1} \cdots i_{n}}$ and $\bar{r}_{i_{1} \cdots i_{n}}$ respectively,

(2) int $\underline{B}_{i_{1} \cdots i_{n}} \cap$ int $\underline{B}_{i_{1}^{\prime} \cdots i_{n}^{\prime}}=\emptyset$ if $\left(i_{1} \cdots i_{n}\right) \neq\left(i_{1}^{\prime}, \ldots, i_{n}^{\prime}\right)$, where int $R$ denotes the interior of the set $R$,

(3) there exist two sequences of numbers

$$
\underline{\lambda}_{i, n}=\lambda_{i} \exp \left(\underline{a}_{i, n}\right), \quad \bar{\lambda}_{i, n}=\lambda_{i} \exp \left(\bar{a}_{i, n}\right)
$$

such that $\underline{r}_{i_{1} \cdots i_{n}}=C_{1} \prod_{j=1}^{n} \underline{\lambda}_{i_{j}, j}$ and $\bar{r}_{i_{1} \cdots i_{n}}=C_{2} \prod_{j=1}^{n} \bar{\lambda}_{i_{j}, j}$, where $0<\lambda_{i}<1, i=$ $1, \ldots, p$ and $C_{1}, C_{2}$ are positive constants,

(4) for $\mu_{\lambda}$-almost every $\omega \in Q$ (where $\lambda=\left(\lambda_{1}, \ldots, \lambda_{p}\right)$ ),

$$
\frac{1}{n} \sum_{j=1}^{n} \underline{a}_{i_{j}, j} \rightarrow 0 \quad \text { and } \quad \frac{1}{n} \sum_{j=1}^{n} \bar{a}_{i_{j}, j} \rightarrow 0 \quad \text { as } n \rightarrow \infty \text {. }
$$

Proposition 7. Let $F$ be the limit set for a Moran-like asymptotic symbolic construction. Then the construction is conditionally regular with the conditionally l-estimating vector equal to $\lambda=\left(\lambda_{1}, \ldots, \lambda_{p}\right)$. Hence $s_{\lambda} \leqq \operatorname{dim}_{H} F$.

Condition (4) in the definition of asymptotic symbolic construction is quite weak; one can obtain more information about the Hausdorff and box dimensions of the limit set if the construction satisfies the following uniform version of (4):

(4a)

$$
\sup _{\left(i_{1} \cdots i_{n}\right)} \frac{1}{n} \sum_{j=1}^{n} \underline{a}_{i_{j}, j} \rightarrow 0 \quad \text { as } n \rightarrow \infty
$$

$$
\sup _{\left(i_{1} \cdots i_{n}\right)} \frac{1}{n} \sum_{j=1}^{n} \bar{a}_{i_{j}, j} \rightarrow 0 \quad \text { as } n \rightarrow \infty
$$


Proposition 8. Let $F$ be the limit set for an Moran-like asymptotic symbolic construction. Assume that the construction satisfies condition (4b). Then $s=s_{i}=$ $\operatorname{dim}_{H} F=\underline{\operatorname{dim}}_{B} F=\overline{\operatorname{dim}}_{B} F$.

Unlike the symbolic construction, the limit set for an asymptotic symbolic construction may have zero Hausdorff measure (even in the case when the construction satisfies the strong asymptotic conditions (a1) and (a2) and the measure $\mu_{\lambda}$ is Gibbs, see Sect. 5, Example 4 and compare with Statement 2 of Proposition 3).

2. Asymptotic one-dimensional symbolic construction. We now consider an asymptotic one-dimensional symbolic construction. This is a one-dimensional geometric construction satisfying Conditions 1 and 2 in the definition of Moran-like asymptotic symbolic constructions and the following conditions:

$\left(3^{\prime}\right)$ there exist two sequences of numbers

$$
\underline{\lambda}_{i, n}=\underline{\lambda}_{i} \exp \left(\underline{a}_{l, n}\right), \quad \bar{\lambda}_{i, n}=\bar{\lambda}_{i} \exp \left(\bar{a}_{l, n}\right)
$$

such that

$$
C_{1} \prod_{j=1}^{n} \underline{\lambda}_{i_{j}, j} \leqq \operatorname{diam}\left(\Delta_{i_{1} \cdots i_{n}}\right) \leqq C_{2} \prod_{j=1}^{n} \bar{\lambda}_{i_{j}, j}
$$

where $0<\underline{\lambda}_{i} \leqq \bar{\lambda}_{i}<1, i=1, \ldots, p$ and $C_{1}, C_{2}>0$ are positive constants,

$\left(4^{\prime}\right)$ for $\mu_{\underline{\underline{\lambda}}}$-almost every $\omega \in Q\left(\right.$ where $\left.\underline{\lambda}=\left(\underline{\lambda}_{1}, \ldots, \underline{\lambda}_{p}\right)\right)$,

$$
\frac{1}{n} \sum_{j=1}^{n} \underline{a}_{i, j} \rightarrow 0 \quad \text { as } n \rightarrow \infty .
$$

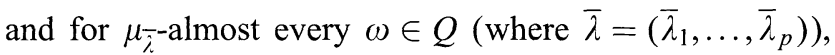

$$
\frac{1}{n} \sum_{j=1}^{n} \bar{a}_{i_{j}, j} \rightarrow 0 \quad \text { as } n \rightarrow \infty \text {. }
$$

Proposition 9. Let $F$ be the limit set for an asymptotic one-dimensional symbolic construction. Then the construction is conditionally regular with the conditionally l-estimating vector $\underline{\lambda}=\left(\underline{\lambda}_{1}, \ldots, \underline{\lambda}_{p}\right)$. Hence $s_{\underline{\lambda}} \leqq \operatorname{dim}_{H} F$.

As in Proposition 8, one can obtain more information about the Hausdorff and box dimensions of the limit set if the construction satisfies the uniform conditions (4a) and (4b).

Proposition 10. Let $F$ be the limit set for an asymptotic one-dimensional symbolic construction. Assume that the construction satisfies condition (4b). Then $s_{\text {L }} \leqq$ $\operatorname{dim}_{H} F \leqq \underline{\operatorname{dim}}_{B} F \leqq \overline{\operatorname{dim}}_{B} F \leqq s_{\bar{i}}$.

3. Random symbolic construction. We now consider a random version of the symbolic construction. In this case the sizes of the basic sets are chosen randomly with respect to some stationary ergodic distribution. We will use an ergodic theorem in [BFKO] to reduce the study of these constructions to the asymptotic Moran constructions. 
A random symbolic construction is defined by

(1) a stochastic vector process $(\Lambda, \mathfrak{F}, v)$ with $\Lambda=\left\{\vec{\lambda}=\left(\underline{\lambda}_{i, n}, \bar{\lambda}_{i, n}\right), i=1, \ldots, p\right.$ and $n \in \mathbb{N}\}$, where $0<\alpha \leqq \underline{\lambda}_{j, n} \leqq \bar{\lambda}_{j, n} \leqq \beta<1$, $\mathbb{F}$ is the $\sigma$-algebra of Borel sets in $\Lambda$, and $v$ is an arbitrary stationary, shift-invariant ergodic Borel probability measure on $\Lambda$,

(2) a compact set $Q \subset \Sigma_{p}^{+}$invariant under the shift (i.e., $\sigma(Q)=Q$ ) such that $\sigma \mid Q$ is topologically transitive,

(3) for $v$-almost every $\vec{\lambda} \in \Lambda$, a family of sets $\left\{\Delta_{i_{1} \cdots i_{n}}(\vec{\lambda})\right\} \subset \mathbb{R}^{n}$ for $i_{j}=$ $1,2, \ldots, p$, where the $n$-tuple $\left(i_{1} \cdots i_{n}\right)$ is admissible with respect to $Q$ which satisfy

$$
\underline{B}_{i_{1} \cdots i_{n}}(\vec{\lambda}) \subset \Delta_{i_{1} \cdots i_{n}}(\vec{\lambda}) \subset \bar{B}_{i_{1} \cdots i_{n}}(\vec{\lambda}),
$$

where $\underline{B}_{i_{1} \cdots i_{n}}(\vec{\lambda})$ and $\bar{B}_{i_{1} \cdots i_{n}}(\vec{\lambda})$ are closed balls having radii

$$
\underline{r}_{i_{1} \cdots i_{n}}(\vec{\lambda})=C_{1} \prod_{j=1}^{n} \underline{\lambda}_{i_{j}, j}(\vec{\lambda}), \quad \bar{r}_{i_{1} \cdots i_{n}}(\vec{\lambda})=C_{1} \prod_{j=1}^{n} \bar{\lambda}_{i_{j}, j}(\vec{\lambda}),
$$

(4) int $\underline{B}_{i_{1} \cdots i_{n}}(\vec{\lambda}) \cap \operatorname{int} \underline{B}_{i_{1}^{\prime} \cdots i_{n}^{\prime}}(\vec{\lambda})=\emptyset$ if $\left(i_{1} \cdots i_{n}\right) \neq\left(i_{1}^{\prime}, \ldots, i_{n}^{\prime}\right)$.

For every $\vec{\lambda} \in \Lambda$, the limit set

$$
F(\vec{\lambda})=\bigcap_{n=1}^{\infty} \bigcup_{\substack{\left(1_{1} \cdots i_{n}\right) \\ \text { admissible }}} \Delta_{i_{1} \cdots i_{n}}(\vec{\lambda})
$$

is a perfect, nowhere dense, totally disconnected set.

The following lemma describes the limiting behavior of the numbers $\underline{\lambda}_{l, n}, \bar{\lambda}_{i, n}$ in the random symbolic construction:

Lemma 1. Let $F$ be the limit set specified by a random symbolic geometric construction. Then there are numbers $\underline{\lambda}_{l}, \bar{\lambda}_{i}, 0<\underline{\lambda}_{l} \leqq \bar{\lambda}_{i}<1, i=1, \ldots, p$ such that for $v$-almost every $\vec{\lambda} \in \Lambda$ the following limits exist:

(1) for $\mu_{\underline{\lambda}}$-almost every sequence $\left(i_{1} i_{2} \cdots\right) \in Q(\vec{\lambda})$,

$$
\lim _{n \rightarrow \infty} \frac{1}{n} \sum_{k=1}^{n} \underline{a}_{i_{k}, k}=0
$$

(2) for $\mu_{-}$-almost every sequence $\left(i_{1} i_{2} \cdots\right) \in Q(\vec{\lambda})$,

$$
\lim _{n \rightarrow \infty} \frac{1}{n} \sum_{k=1}^{n} \bar{a}_{i_{k}, k}=0
$$

where $\underline{a}_{i, j}=\log \left(\frac{\underline{\underline{i}}_{i, j}}{\underline{\hat{\lambda}}_{l}}\right), \bar{a}_{l, j}=\log \left(\frac{\overline{\bar{\lambda}}_{\bar{\lambda}_{l}}}{\overline{\hat{\lambda}}_{l}}\right), \underline{\lambda}=\left(\underline{\lambda}_{1}, \ldots, \underline{\lambda}_{p}\right), \bar{\lambda}=\left(\bar{\lambda}_{1}, \ldots, \bar{\lambda}_{p}\right)$. and 9.

The next statement immediately follows from Lemma 1 and Propositions 7

Proposition 11. Let $F$ be the limit set specified by a random symbolic construction. Assume that either the construction is one-dimensional or $\underline{\lambda}_{i}=\bar{\lambda}_{i}$ for $i=1, \ldots, p$. Then for $v$-almost every $\vec{\lambda} \in \Lambda$,

$$
s_{\underline{\underline{\lambda}}} \leqq \operatorname{dim}_{H} F(\vec{\lambda}) .
$$




\section{Generating Maps and Codings}

One particular but important case of a geometric construction is when the sets $\Delta_{i_{1}} \cdots i_{n}$ are given by

$$
\Delta_{i_{1} \cdots i_{n}}=h_{i_{1}} \circ h_{i_{2}} \circ \cdots \circ h_{i_{n}}(\Delta)
$$

where $h_{1}, \ldots, h_{p}: \Delta \rightarrow \Delta$ are contraction maps, i.e., $d\left(h_{i}(x), h_{i}(y)\right) \leqq L_{i} d(x, y)$ with $L_{i}<1$ and $x, y \in \Delta$ (a ball in $\mathbb{R}^{n}$ ), where $d$ denotes Euclidean distance. Most of the results in the literature in dimension theory require that the process be described in this way. We stress that this is a very special case and that the following situations can (and do) occur:

(1) The construction can not be described by any continuous maps, i.e., there are no continuous maps satisfying (8). This can occur if the boundary of a set $\Delta_{i_{1} \cdots i_{n}}$ is fractal. In the one-dimensional case the maps $h_{j}$ always exist and are continuous, so the above mentioned pathology does not occur. In this case the maps are well defined by

$$
h_{j}\left(\partial \Delta_{i_{1} \cdots i_{n}}\right)=\partial \Delta_{i_{1} \cdots i_{n} j}
$$

for any admissible sequence $\left(i_{1} \cdots i_{n} j\right)$, where $\partial \Delta_{l_{1} \cdots l_{n}}$, denotes the boundary of the set $\Delta_{i_{1} \cdots i_{n} j}$.

(2) There are continuous maps satisfying (8) that are not Lipschitz. One obstruction is that the boundary of a set $\Delta_{l_{1} \cdots l_{n}}$ can be a continuous but not Lipschitz image of $\partial \Delta$. In the one-dimensional case, this obstruction cannot occur. However, one can construct a one-dimensional example where the process is defined by continuous but not Lipschitz maps. Namely, there exists a simple geometric construction on [0,1] with $p=2, \underline{\lambda}_{1}=\bar{\lambda}_{1}=\lambda_{1}, \underline{\lambda}_{2}=\bar{\lambda}_{2}=\lambda_{2}, 0<\lambda_{1}<\lambda_{2}<1$ and the locations of the intervals $\Delta_{i_{1} \cdots i_{n}}$ are such that the map $h_{1}$ is not Lipschitz. Choose basic sets such that

$$
\frac{d(\underbrace{\Delta_{1 \cdots 1,}^{1 \cdots}}_{n_{k}} \Delta_{n_{n_{k}-1}}^{\underbrace{1 \ldots 1,}_{n_{k}-1,2},})}{d\left(\Delta_{n_{k}-1}^{\Delta_{1, \ldots, 1}, \Delta_{1,2,1}}\right)} \stackrel{\Delta_{k} \rightarrow \infty}{\longrightarrow} \infty .
$$

(3) There are Lipschitz maps satisfying (8) but these maps are not contractions. To see this, choose intervals $\Delta_{1}, \Delta_{2}$ and maps $h_{1}, h_{2}$ such that $\Delta_{11}=h_{1}\left(\Delta_{1}\right)$, $\Delta_{21}=h_{1}\left(\Delta_{2}\right)$ and $d\left(\Delta_{1}, \Delta_{2}\right) \ll d\left(\Delta_{11}, \Delta_{21}\right)$. Then the map $h_{1}$ is not a contraction.

(4) There are contraction maps satisfying (8) whose inverse maps are not Lipschitz. The Lipschitz constants for the inverse maps depend on the gaps between the sets $\Delta_{i_{1} \cdots i_{n}}$. To see this, choose intervals $\Delta_{i_{1} \cdots l_{n}}$ such that

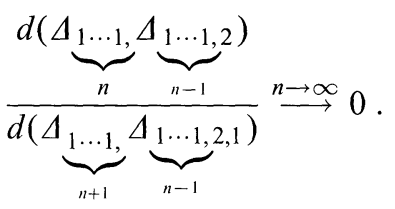

Then the inverse of the map $h_{1}$ is not Lipschitz.

(5) There are contraction maps satisfying (8) whose inverse maps are Lipschitz but the maps are not similarities (i.e., $d\left(h_{i}(x), h_{i}(y)\right)=L_{i} d(x, y)$ for all $\left.x \in \Delta\right)$.

As we saw in case (4) the Lipschitz constants for the inverse maps may depend on the gaps between the sets $\Delta_{i_{1} \cdots i_{n}}$. For this reason, even if the process can be 
described using contraction maps with Lipschitz inverses, they may be of no use in estimating the Hausdorff dimension of the limit set.

Let $F$ be the limit set specified by a symbolic construction. Assume that the construction satisfies the separation condition $\left(5^{\prime}\right)$. In this case the coding map $\chi$ is injective. Define the map $G: F \rightarrow F$ by $G(x)=\chi \circ \sigma \circ \chi^{-1}(x)$. It is easy to see that $G$ is a continuous endomorphism such that the set $G^{-1}(x), x \in F$ consists of finitely many points. If $\mu$ is a $\sigma$-invariant measure on $Q$ then its push forward measure $m=\chi^{*} \mu$ is $G$-invariant and has the same ergodic properties as $\mu$. If the construction is given by finitely many contraction maps $h_{k}$ then the map $G$ can be described as

$$
G(x)=h_{k}^{-1}(x) \text { if } x \in \Delta_{k} .
$$

A very interesting question is to find criteria for a set $F$ to be the limit set of a geometric construction such that the induced map $G: F \rightarrow F$ is expanding (see Appendix 5). One may at first believe that the induced maps $G$ for geometric constructions having exponentially large gaps are expanding, but this is easily seen to be false.

\section{More Examples}

\section{1) Sierpiński Gaskets}

a) It is well known that the Hausdorff dimension of the Sierpiński gasket (Fig. 2a) coincides with the box dimension and is $\frac{\log 3}{\log 2}$. This immediately follows from Corollary 2 and Proposition 3 since $\lambda_{1}=\lambda_{2}=\lambda_{3}=\frac{1}{2}$ and $p=3$.

b) Suppose that in the construction of the Sierpiński gasket we forbid all configurations whose codings contain a 1 followed by a 2 (Fig. 2b). The spectral radius of $\left(\begin{array}{lll}1 & 0 & 1 \\ 1 & 1 & 1 \\ 1 & 1 & 1\end{array}\right)$ is $\frac{3+\sqrt{5}}{2}$. Hence by Proposition 1 and Corollary $1, \operatorname{dim}_{H} F=\frac{\log \left(\frac{3+\sqrt{5}}{2}\right)}{\log 2} \approx$ 1.38848 .

c) A simple construction of the Sierpiński gasket with $\lambda_{i}=\frac{1}{3} \quad$ for $i=1,2,3$ is illustrated in Fig. $2 \mathrm{c}$ ). The sets $\Delta_{i_{1} \cdots i_{n}}$ are asymptotically congruent to the corresponding triangles in the usual constructions and possess wiggly boundaries that become asymptotically straight. As long as the approximation is sufficiently fast and uniform, such that the construction satisfies the hypotheses of Proposition 8,

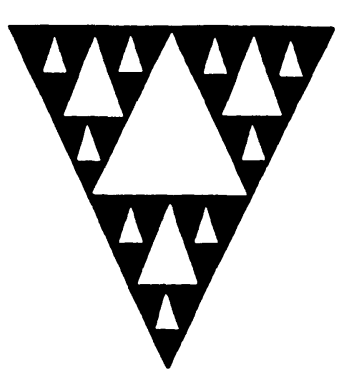

$\mathbf{a}$

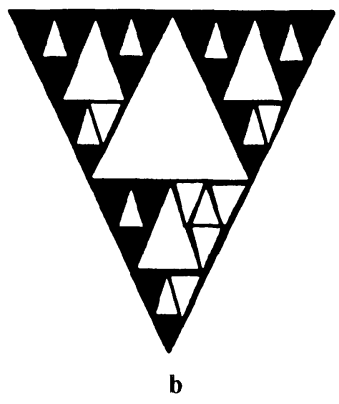

Fig. 2a-c. Sierpiński Gaskets

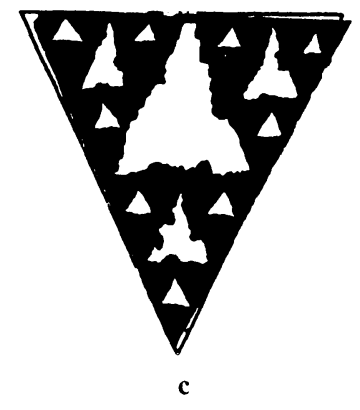


then the Hausdorff dimension of the limit set is $\frac{\log 3}{\log 2}$. One can even choose the sets $\Delta_{i_{1} \ldots i_{n}}$ in the construction to have fractal boundaries.

2) General Smale-Williams solenoid. Let $P$ be a solid torus embedded in $\mathbb{R}^{3}$. We represent points on $P$ by means of coordinates $(\theta, r, s)$, where $\theta \in S^{1}$ the unit circle, $-1 \leqq r, s \leqq 1$ such that $r^{2}+s^{2} \leqq 1$. The point $x$ with coordinates $(\theta, r, s)$ belongs to the plane orthogonal to the core of the torus through the point $\theta \in S^{1}$ having position $(r, s)$ relative to the standard frame $\left(e_{1}, e_{2}\right)$. We define a mapping $f: P \rightarrow P$ by

$$
f(\theta, r, s)=\left(2 \theta, \lambda_{1} r+\varepsilon \cos \theta, \lambda_{2} s+\varepsilon \sin \theta\right),
$$

where $p>0$ is an integer, $\varepsilon$ is a small positive constant and $0<\lambda_{1}, \lambda_{2}<1$. The image $f(P)$ is contained in $P$ and wraps twice around $P$. See Fig. 3a). The set $\Delta=\bigcap_{n=1}^{\infty} f^{n}(P)$ is called a solenoid. See [Sh] for more details and nice pictures.

Let $D_{\theta}$ be the section of $P$ determined by the plane perpendicular to the core at $\theta$. The set $\Delta_{\theta}=\Delta \cap D_{\theta}$ is the Cantor-like set obtained by the simple geometric construction with $\underline{\lambda}_{1}=\lambda_{1}$ and $\bar{\lambda}_{i}=\lambda_{2}$ for $i=1,2$. See Fig. $3 \mathrm{~b}$. Since the basic sets in the construction of $\Delta_{\theta}$ are rectangles, it follows from Proposition 4 that

$$
\frac{\log 2}{\log \left(\frac{1}{\lambda_{1}}\right)} \leqq \operatorname{dim}_{H} \Delta_{\theta} \leqq \frac{\log 2}{\log \left(\frac{1}{\lambda_{2}}\right)} .
$$

Applying Marstrand's theorem [F1], we obtain

$$
1+\frac{\log 2}{\log \left(\frac{1}{\lambda_{1}}\right)} \leqq \operatorname{dim}_{H} \Delta .
$$

A very simple argument $[\mathrm{F} 1]$ shows that $\operatorname{dim}_{H} \Delta \leqq 1+\frac{\log 2}{\log \left(\frac{1}{i_{2}}\right)}$.

If $\lambda_{1}=\lambda_{2}=\lambda$, we have that $\operatorname{dim}_{H} \Delta_{\theta}=\frac{\log 2}{\log \left(\frac{1}{j}\right)}$ and $\operatorname{dim}_{H} \Delta=\frac{\log 2}{\log \left(\frac{1}{i}\right)}+1$, which is the result obtained by Falconer [F1].

3) Geometric construction with rectangles in the plane. The following simple example illustrates the fact that the regularity of the construction depends not only on
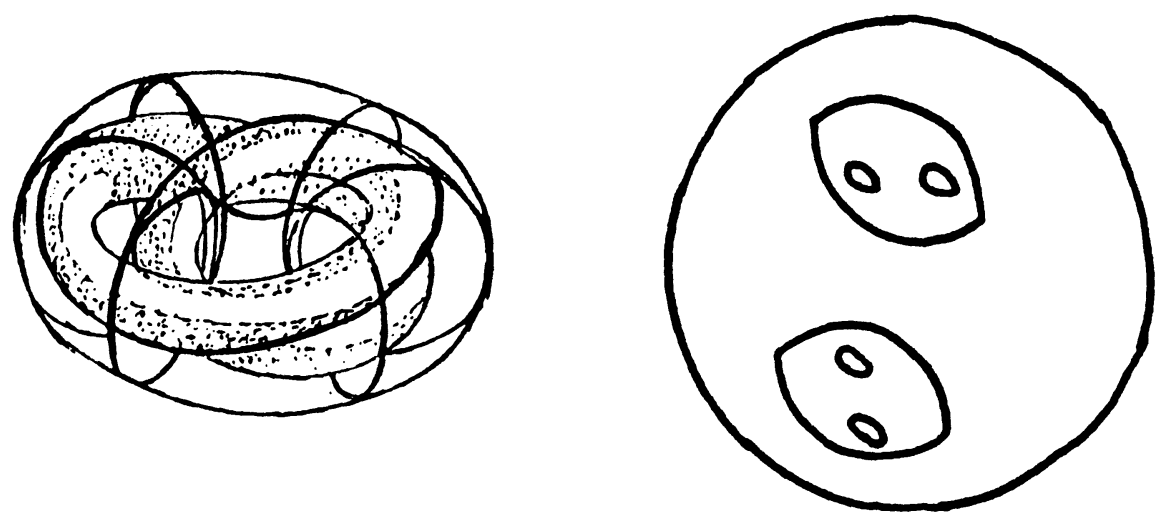

Fig. 3. Smale-Williams Solenoid a), b) 
the sizes and shapes of the basic sets but also may depend on their spacing. Consider the two similarity constructions where all the basic sets at step $n$ are congruent rectangles with width $\underline{\lambda}^{n}$ and length $\bar{\lambda}^{n}$. The rectangles are stacked horizontally in the first construction and vertically in the second construction. The limit sets of both constructions are one-dimensional Cantor sets. The first has Hausdorff dimension $\frac{\log 2}{-\log \bar{\lambda}}$ and the second has Hausdorff dimension $\frac{\log 2}{-\log \underline{\lambda}}$. The first construction is regular with 1-estimating vector $\gamma=(\bar{\lambda}, \bar{\lambda})$. The vector $(\bar{\lambda}, \bar{\lambda})$ is not l-estimating for the second construction but is l-estimating for the first construction.

4) A simple asymptotic construction. This example shows that the second statement of Proposition 3 may fail for an asymptotic construction, i.e., the measure of the limit set may be zero.

Let $p=2$ and suppose $\lambda_{i, n}=\lambda_{n}=\lambda \exp \left(a_{n}\right)$ for $i=1,2$. There exists a geometric construction on the line with disjoint basic sets $\Delta_{i_{1} \cdots i_{n}}$ satisfying

$$
\operatorname{diam}\left(\Delta_{i_{1} \cdots i_{n}}\right)=\prod_{j=1}^{n} \lambda_{i_{j}, j}=\lambda^{n} \exp \left(A_{n}\right),
$$

where $A_{n}=\sum_{k=1}^{n} a_{k}$. Let $F$ be the limit set. For fixed $n$, the sets $\left\{\Delta_{\iota_{1} \cdots i_{n}}\right\}$ give a cover of $F$ with

$$
\sum_{i_{1} \cdots l_{n}} \operatorname{diam}\left(\Delta_{i_{1} \cdots i_{n}}\right)^{s}=2^{n} \lambda^{n s} \exp \left(s A_{n}\right)=\exp \left(s A_{n}\right),
$$

where $s=\frac{\log 2}{\log \left(\frac{1}{1}\right)}$. There is now a trichotomy:

(1) if $\sum_{k=1}^{\infty} a_{k}<\infty$, then one can easily show that the geometric process satisfies conditions a1) and a2), and by Proposition $8, s=\operatorname{dim}_{H} F=\operatorname{dim}_{B} F=\frac{\log 2}{\log \left(\frac{1}{1}\right)}$,

(2) if $\sum_{k=1}^{\infty} a_{k}=\infty$, then one can show that $m_{H}(s, F)=\infty$,

(3) if $\sum_{k=1}^{\infty} a_{k}=-\infty$, then $m_{H}(s, F)=0$.

The sequence $\left\{a_{k}\right\}$ satisfies the condition in the definition of asymptotic construction if and only if $\frac{1}{n} A_{n} \rightarrow 0$. For example, choose $a_{n}=\frac{1}{n}$ in case (2) and $a_{n}=-\frac{1}{n}$ in case (3). One can show that $s$ is still the Hausdorff dimension of the limit set $F$. The mass distribution principle does not hold in case (3) since otherwise we would have $m_{H}(s, F)>0$.

We now construct gauge functions for certain sequences $\left\{a_{k}\right\}$, where $\sum_{k=1}^{\infty} a_{k}$ $=-\infty$. Let $A_{n}=\sum_{k=1}^{n} a_{k}$. We seek a function $h(t)$ such that $0<2^{n} h\left(\lambda^{n} \exp \left(A_{n}\right)\right)<$ $\infty$. We will find $h(t)$ in the form $h(t)=t^{s} \exp (\phi(t))$. Then we would have $-\infty<$ $s A_{n}+\phi\left(\lambda^{n} \exp \left(A_{n}\right)\right)<\infty$. Define $t=\lambda^{n} \exp \left(A_{n}\right)$. Since $\lim _{n \rightarrow \infty} \frac{A_{n}}{n}=0$, then $t \asymp$ $\lambda^{n}$, and hence we can set $\phi(t)=-s A_{\frac{\log t}{\log t}}$.

If $a_{n}=-\frac{1}{n}$ then $A_{n}=O(-\log n)$ and $\phi(t)=\log \left(\frac{\log t}{\log \lambda}\right)$. If $a_{n}=-\frac{1}{n \log n}$ then $A_{n}=O(-\log (\log n))$ and $\phi(t)=\log \left(\log \left(\frac{\log t}{\log \lambda}\right)\right)$. More generally, if $a_{n}=-\frac{1}{n \log ^{(i)} n}$, where $\log ^{(i)} n$ denotes the $i$-fold composition of $\log n$, then $A_{n}=O\left(-\log ^{(i+1)} n\right)$ and hence $\phi(t)=\log ^{(i+1)}\left(\frac{\log t}{\log \lambda}\right)$. We can thus obtain gauge functions with arbitrarily many logs from this basic construction. For these different sequences, the coefficients $\lambda_{n}=\lambda \exp \left(a_{n}\right)$ converge to $\lambda$, but of course with different speeds, and hence, require different gauge functions. 
5) Random version of Example 4. The following example is a special case of random constructions that was pointed out to the authors by Peres. We consider the construction in Example 4 where the numbers $\left\{a_{n}\right\}$ are independent and identically distributed random variables on the interval $\infty<a \leqq a_{n} \leqq b<\log \left(\frac{1}{2}\right)$ having mean 0 . If we define the random variable $A_{n}=\sum_{k=1}^{n} a_{k}$, then the law of the iterated logarithm implies that $\lim \inf A_{n}=-\infty$. The law of large numbers shows that $\frac{A_{n}}{n} \rightarrow 0$. It immediately follows from a simple calculation as in Example 4 that $s=\operatorname{dim}_{H} F=\frac{\log 2}{-\log \lambda}$ and $m_{H}(s, F)=0$.

6) Simple geometric construction with rectangles having limit set $F$ for which $\operatorname{dim}_{H} F<\underline{\operatorname{dim}}_{B} F<\overline{\operatorname{dim}}_{B} F$. We describe a simple construction with rectangles in $\mathbb{R}^{2}$ with $p=2, \underline{\lambda}_{1}=\underline{\lambda}_{2}=\underline{\lambda}, \bar{\lambda}_{1}=\bar{\lambda}_{2}=\bar{\lambda}, 0<\underline{\lambda}<\bar{\lambda}<\frac{1}{3}$ such that the limit set $F$ satisifes

$$
\operatorname{dim}_{H} F=\frac{\log 2}{-\log \underline{\lambda}}, \quad \underline{\operatorname{dim}}_{B} F=\gamma \frac{\log 2}{-\log \underline{\lambda}}, \quad{\operatorname{dim}_{B} F} F=\frac{\log 2}{-\log \bar{\lambda}},
$$

where $\gamma \in(1, \alpha)$ is an arbitrary number and $\alpha=\frac{\log \hat{\lambda}}{\log \overline{\bar{\lambda}}}>1$.

Let $n_{0}=0$ and for $k=0,1,2, \ldots$, let $n_{k+1}=\left[\alpha n_{k}\right]$ and $\beta_{k}=2^{(\gamma-\alpha) n_{3 k+1}}$. In order to describe the $n^{\text {th }}$ step of the construction we use the basic types of spacings: vertical stacking (a) and horizontal staking (B). See Fig. 4.

(1) We start with two horizontally stacked rectangles. During steps $n_{3 k}<n \leqq$ $n_{3 k+1}$ we use (b).

(2) We begin with $2^{n_{3 k+1}}$ rectangles. Choose $\beta_{k}$ percent of these rectangles arbitrarily and paint them blue; paint the others green. During steps $n_{3 k+1}<n \leqq n_{3 k+2}$, we use (b) in all blue rectangles and use (a) in all green rectangles.

(3) During steps $n_{3 k+2}<n \leqq n_{3 k+3}$, we use (a) in all blue rectangles and use (b) in all green rectangles.

(4) Repaint all $2^{n_{3 k+3}}$ rectangles white; repeat steps 1 to 4 .

The collection of rectangles at the $n^{\text {th }}$ step contains $2^{n}$ rectangles each with vertical and horizontal sides of size $\underline{\lambda}^{n} \times \bar{\lambda}^{n}$ (the size in the vertical direction is $\underline{\lambda}^{n}$

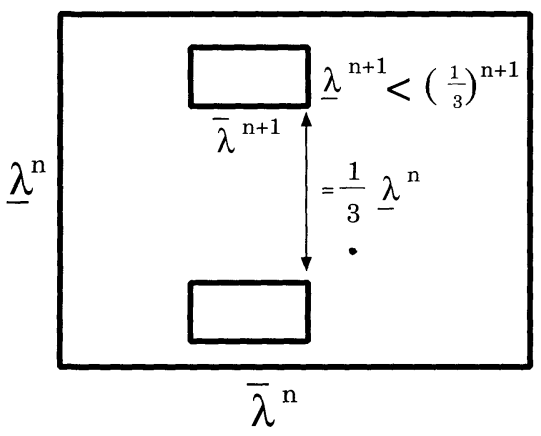

a

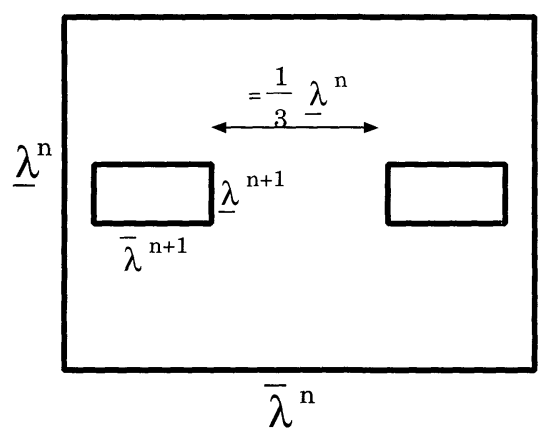

b

Fig. 4. a vertical stacking, b horizontal stacking 
and the size in the horizontal direction is $\bar{\lambda}^{n}$ ). Any two subrectangles on step $n+1$ that are contained in the same rectangle at step $n$ are stacked either horizontally or vertically and the distance between them is $\frac{1}{3} \underline{\lambda}^{n}$. The projections of any two distinct rectangles at step $n$ onto the two coordinate axes either coincide or are disjoint.

Let us note that one can effect this geometric construction by a sequence of affine maps $\left\{h_{i, n}\right\}$ such that each basic set $\Delta_{i_{1} \cdots i_{n}}=h_{i_{1}, n} \circ \cdots \circ h_{i_{n}, n}([0,1])$ and the induced map $G$ on the limit set $F$ is expanding (see Appendix 5).

We now calculate the Hausdorff dimension and the lower and upper box dimensions of the limit set $F$.

a) Calculation of Upper and Lower Box Dimensions. Choose $\varepsilon>0$. There exists a unique integer $n>0$ such that $\bar{\lambda}^{n+1}<\varepsilon \leqq \bar{\lambda}^{n}$. There are three possible cases and we explicitly compute $N_{\varepsilon}(F)$ in each case. Denote by

$$
A_{n}=\frac{\log N_{\varepsilon}(F)}{-\log \bar{\lambda}^{n}} .
$$

Case 1. We have $n_{3 k} \leqq n<n_{3 k+1}$. In this case $N(\varepsilon)=2^{n}$ and hence

$$
A_{n}=\frac{\log 2}{-\log \bar{\lambda}} .
$$

Case 2. We have $n_{3 k+1} \leqq n<n_{3 k+2}$. In this case $N_{\varepsilon}(F)=N_{\varepsilon}^{\text {blue }}(F)+N_{\varepsilon}^{\text {green }}(F)$, where $N_{\varepsilon}^{\text {blue }}(F)$ and $N_{\varepsilon}^{\text {green }}(F)$ are the numbers of $\varepsilon$-balls in the optimal cover that have non-empty intersection with respectively blue and green rectangles at step $n$. It follows that

$$
N_{\varepsilon}(F)=\beta_{k} 2^{n}+\left(1-\beta_{k}\right) 2^{n_{3 k+1}} .
$$

One can see that for sufficiently large $k$ (for which $\beta_{k} \leqq \frac{1}{2}$ ) we have

$$
N_{\varepsilon}(F) \leqq 2\left(2^{(\gamma-\alpha) n_{3 k+1}} 2^{\alpha n_{3 k+1}+n-n_{3 k+2}}+2^{n_{3 k+1}}\right)=2\left(2^{\gamma n_{3 k+1}} 2^{n-n_{3 k+2}}+2^{n_{3 k+1}}\right),
$$

and

$$
N_{\varepsilon}(F) \geqq \frac{1}{2}\left(2^{(\gamma-\alpha) n_{3 k+1}} 2^{\alpha n_{3 k+1}+n-n_{3 k+2}}+2^{n_{3 k+1}}\right)=\frac{1}{2}\left(2^{\gamma n_{3 k+1}} 2^{n-n_{3 k+2}}+2^{n_{3 k+1}}\right) .
$$

(1) Suppose $n=n_{3 k+1}$. Then

$$
\lim _{n \rightarrow \infty} A_{n}=\frac{\log 2}{-\log \bar{\lambda}}
$$

(2) Suppose $n=n_{3 k+2}-1$. Then

$$
\lim _{n \rightarrow \infty} A_{n}=\frac{\gamma \log 2}{-\alpha \log \bar{\lambda}}=\frac{\gamma \log 2}{-\log \underline{\lambda}} .
$$

(3) Suppose $n_{3 k+1}<n<n_{3 k+2}$. We wish to show that

$$
\lim \inf A_{n} \geqq \frac{\gamma \log 2}{-\log \underline{\lambda}}=\frac{\gamma \log 2}{-\alpha \log \bar{\lambda}} .
$$


It is enough to establish that

$$
2^{\gamma n_{3 k+1}} 2^{n-n_{3 k+2}}+2^{n_{3 k+1}} \geqq 2^{\frac{i n}{\alpha}}
$$

This is an easy calculus exercise.

Case 3. We have $n_{3 k+2} \leqq n<n_{3 k+3}$. In this case

$$
N_{\varepsilon}(F)=N_{\varepsilon}^{\text {blue }}(F)+N_{\varepsilon}^{\text {green }}(F)=\beta_{k} 2^{n_{3 k+2}}+\left(1-\beta_{k}\right) 2^{n} .
$$

It is easy to see that for sufficiently large $k$ (for which $\beta_{k} \leqq \frac{1}{2}$ ) we have that

$$
N_{\varepsilon}(F) \leqq 2\left(2^{(\gamma-\alpha) n_{3 k+1}} 2^{\alpha n_{3 k+1}}+2^{n}\right)=2\left(2^{n}+2^{\gamma n_{3 k+1}}\right)
$$

and

$$
N_{\varepsilon}(F) \geqq \frac{1}{2}\left(2^{(\gamma-\alpha) n_{3 k+1}} 2^{\alpha n_{3 k+1}}+2^{n}\right)=\frac{1}{2}\left(2^{n}+2^{\gamma n_{3 k+1}}\right)
$$

(1) Suppose $n=n_{3 k+2}$. Then

$$
\lim _{n \rightarrow \infty} A_{n}=\frac{\log 2}{-\log \bar{\lambda}} .
$$

(2) Suppose $n=n_{3 k+3}-1$. Then

$$
\lim _{n \rightarrow \infty} A_{n}=\frac{\log 2}{-\log \bar{\lambda}}
$$

(3) Suppose $n_{3 k+2}<n<n_{3 k+3}$. We wish to show that

$$
\liminf _{n \rightarrow \infty} A_{n} \geqq \frac{\log 2}{-\log \underline{\lambda}}
$$

It is certainly enough to establish that

$$
2^{n}+2^{\gamma n_{3 k+1}} \geqq 2^{n}
$$

This is obvious.

It follows that $\overline{\operatorname{dim}}_{B} F \geqq \frac{\log 2}{-\log \bar{\lambda}}$. Combining this with Proposition 4, we conclude that $\overline{\operatorname{dim}}_{B} F=\frac{\log 2}{-\log \bar{\lambda}}$. It also follows that $\underline{\operatorname{dim}}_{B} F \geqq \gamma \frac{\log 2}{-\log \underline{\lambda}}$. Since $\lim A_{n_{3 k+1}}=\gamma \frac{\log 2}{-\log \underline{\underline{\lambda}}}$, we conclude that $\underline{\operatorname{dim}}_{B} F=\gamma \frac{\log 2}{-\log \underline{\underline{\lambda}}}$. 
b) Calculation of Hausdorff Dimension. Given $\varepsilon>0$, choose $k>0$ such that $\underline{\lambda}^{n_{3 k+1}} \leqq \varepsilon$. Consider the covering of $F$ consisting of green rectangles for $n=n_{3 k+1}$ and blue rectangles for $n=n_{3 k+2}$.

Consider a green rectangle $\Delta_{i_{1} \cdots i_{n_{3 k+1}}}$. By construction, the intersection $A=$ $\Delta_{i_{1} \cdots i_{n_{3 k+1}}} \cap F$ is contained in $2^{n_{3 k+2}-n_{3 k+1}}$ small green rectangles corresponding to $n=n_{3 k+2}$. These rectangles are vertically aligned and have size $\underline{\lambda}^{n_{3 k+2}} \times \bar{\lambda}^{n_{3 k+2}}$. Since $\bar{\lambda}^{n_{3 k+2}}=\bar{\lambda}^{\left[\alpha n_{3 k+1}\right]+2} \leqq \frac{1}{3} \underline{\lambda}^{n_{3 k+1}}$, the $\left(1-\beta_{k}\right) 2^{n_{3 k+1}}$ green rectangles in the construction of $F$ are each contained in a green square of size $\underline{\lambda}^{n_{3 k+1}}$.

Now consider a blue rectangle $\Delta_{i_{1} \cdots l_{n_{3 k+1}}}$. By our construction, the intersection $B=\Delta_{i_{1} \cdots i_{n_{3 k+2}}} \cap F$ is contained in $2^{n_{3 k+3}-n_{3 k+2}}$ small blue rectangles corresponding to $n=n_{3 k+3}$. They are vertically aligned and have size $\underline{\lambda}^{n_{3 k+3}} \times \bar{\lambda}^{n_{3 k+3}}$. Since $\bar{\lambda}^{n_{3 k+3}} \leqq$ $\frac{1}{3} \underline{\lambda}^{n_{3 k+2}}$, the $\beta_{k} 2^{n_{3 k+1}} 2^{n_{3 k+2}-n_{3 k+1}}=\beta_{k} 2^{n_{3 k+2}}$ blue rectangles in the construction of $F$ are each contained in a blue square of size $\underline{\lambda}^{n^{3 k+2}}$.

The collection of green and blue squares comprises a covering $\mathfrak{G}=\left\{U_{i}\right\}$ of $F$ such that

$$
\sum_{U_{i} \in \mathfrak{F}}\left(\operatorname{diam} U_{i}\right)^{s}=\left(1-\beta_{k}\right) 2^{n_{3 k+1}}\left(\sqrt{2} \underline{\lambda}^{n_{3 k+1}}\right)^{s}+\beta_{k} 2^{n_{3 k+2}}\left(\sqrt{2} \underline{\lambda}^{n_{3 k+2}}\right)^{s}>1>0
$$

if $s=\frac{\log 2}{-\log \underline{\underline{\lambda}}}$. This implies that $\operatorname{dim}_{H} F \leqq \frac{\log 2}{-\log \bar{\lambda}}$. On the other hand, by Proposition 4 , we know that $\operatorname{dim}_{H} F \geqq \frac{\log 2}{-\log \underline{\underline{\lambda}}}$, hence $\operatorname{dim}_{H} F=\frac{\log 2}{-\log \underline{\underline{\lambda}}}$.

7) Example where diameters of inscribed balls in basic sets is not an l-estimating vector. Let $\gamma_{1}, \gamma_{2}, \gamma_{3}, \lambda$ be any numbers in $(0,1)$ and let $A\left(\gamma_{i}\right)$ denote a simple geometric construction on the interval $[0,1] \times\{i-2\}, i=1,2,3$ with $2^{n}$ basic sets of size $\gamma_{i}^{n}$ at step $n$. We wish to use these three one-dimensional constructions to define a simple geometric construction in the square $[0,1] \times[-1,1]$. Since the $2^{n}$ intervals at step $n$ in each of the one-dimensional constructions are clearly ordered, we may refer to the $i^{\text {th }}$ subinterval at step $n, 1 \leqq i \leqq 2^{n}$ of these constructions. Consider the $2^{n}$ polygons in $[0,1] \times[-1,1]$ having six vertices which consist of the two endpoints of the $i^{\text {th }}$ subinterval at step $n$ for all three constructions. We define the $2^{n}$ basic sets of our construction at step $n$ by intersecting these $2^{n}$ polygons with the rectangle $[0,1] \times\left[-\lambda^{n}, \lambda^{n}\right]$. See Fig. 5 .

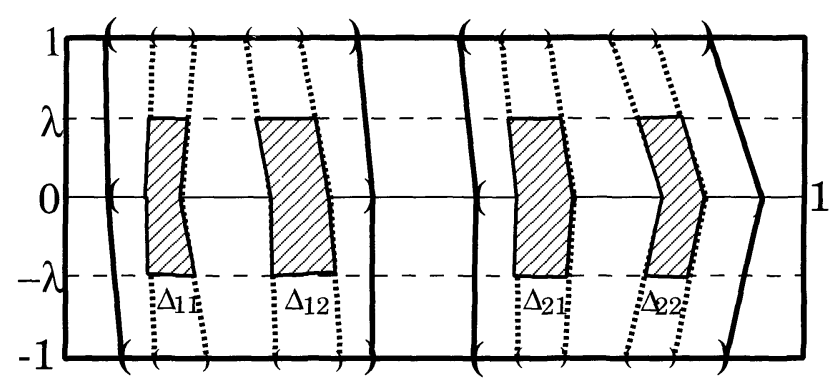

Fig. 5. 
It is easy to see that the limit set $F$ of this construction coincides with the limit set of the construction $A\left(\gamma_{2}\right)$. Hence $\operatorname{dim}_{H} F=\frac{\log 2}{-\log \gamma_{2}}$ and does not depend on $\gamma_{1}, \gamma_{3}$ or $\lambda$. If we choose $\gamma_{2}<\gamma_{1}=\gamma_{3}<\lambda$ and $\gamma_{2}<\lambda \gamma_{1}$ or $\gamma_{2}<\lambda \gamma_{3}$, then the inscribed and circumscribed balls of the basic sets at step $n$ have radii $C_{1} \gamma_{1}^{n}$ and $C_{2} \lambda^{n}$, where $C_{1}$ and $C_{2}$ are positive constants that are independent of $n$. Thus these balls cannot be used to determine the Hausdorff dimension of the limit set.

\section{Pointwise Dimension of Measures Concentrated on Cantor-like Sets and the Continuous Version of the Eckmann-Ruelle Conjecture}

6.1. We consider a class of symbolic geometric constructions modeled by a symbolic dynamical system $(Q, \sigma)$ having compact basic sets $\Delta_{i_{1} \cdots i_{n}}$ and satisfying the following conditions:

$$
D\left(C_{1} \prod_{j=1}^{n} \lambda_{i_{j}}\right) \subset \Delta_{i_{1} \cdots i_{n}} \subset D\left(C_{2} \prod_{j=1}^{n} \lambda_{i_{j}}\right),
$$

where $0<\lambda_{i}<1$ for $i=1, \ldots, p, C_{1}, C_{2}$ are positive constants, and $D(r)$ denotes a ball of radius $r$.

(2) $\Delta_{l_{1} \cdots i_{n}} \cap \Delta_{i_{1}^{\prime} \cdots i_{n}^{\prime}}=\emptyset$ if $\left(i_{1} \cdots i_{n}\right) \neq\left(i_{1}^{\prime}, \ldots, i_{n}^{\prime}\right)$.

This class of constructions includes Moran geometric constructions with disjoint basic sets. Although basic sets of these constructions are essentially balls, their topology and geometry may be quite complicated (for example, they may not be connected and their boundaries may be fractal).

Let $F$ be the limit set for the symbolic geometric construction defined above. We formulate a powerful criterion that allows one to estimate the lower and upper pointwise dimensions with respect to a Borel probability measure $v$ on $F$. Given $x \in F$ and $n>0$, consider the unique set $\Delta_{i_{1} \cdots i_{n}}=\Delta_{n}(x)$ that contains the point $x$. Denote

$$
\begin{aligned}
& \underline{d}(x)=\liminf _{n \rightarrow \infty} \frac{\log v\left(\Delta_{n}(x)\right)}{\log \operatorname{diam} \Delta_{n}(x)} ; \\
& \bar{d}(x)=\limsup _{n \rightarrow \infty} \frac{\log v\left(\Delta_{n}(x)\right)}{\log \operatorname{diam} \Delta_{n}(x)} .
\end{aligned}
$$

Theorem 7. Let $F$ be the limit set for the symbolic geometric construction defined above. Then for any probability measure $v$ supported on $F$

(1) $\bar{d}_{v}(x) \leqq \bar{d}(x)$ for all $x \in F$,

(2) $\underline{d}(x) \leqq \underline{d}_{v}(x)$ for $v$-almost all $x \in F$,

(3) If $\underline{d}(x)=\bar{d}(x) \stackrel{\text { def }}{\equiv} d(x)$ for v-almost every $x \in F$, then

$$
\underline{d}_{v}(x)=\bar{d}_{v}(x)=d(x)
$$

for v-almost every $x \in F$.

Remark. Consider a symbolic geometric construction with basic sets satisfying only Condition 1 above (thus we do not require that the basic sets are disjoint). In this case the coding map need not be injective. Let $F^{\prime}$ denote the set of points $x \in F$ such that $\chi^{-1}(x)$ consists of only one point, i.e., where the coding map is injective. 
By replacing the set $F$ by the set $F^{\prime}$ in the proof of Theorem 7 one can show that Statements 1 and 2 hold for all point $x \in F^{\prime}$. For an application to multifractal analysis see [PW2].

The next statement is an immediate corollary of Theorem 7.

Corollary 3. Let $F$ be the limit set for a symbolic geometric construction defined above. Assume that there is a Borel measure $v$ on $F$ such that $\underline{d}(x)=\bar{d}(x) \stackrel{\text { def }}{\equiv} s$ for $v$-almost every $x \in F$. Then $s \leqq \operatorname{dim}_{H} F$.

Using Theorem 7 and the Birkhoff Ergodic Theorem we now show that for the above symbolic constructions, the pointwise dimension of any measure $v$ which is supported on the limit set $F$ exists and is constant almost everywhere, provided the pull back of $v$ under the coding map is a Gibbs measure. We can assume that the potential $\log \psi$ for $v$ satisfies $P(\log \psi)=0$, for if $\log \phi$ is any potential for $v$, let $\log \psi=\log \phi-P(\log \phi)$.

Since the basic sets of the construction are disjoint, the coding map $\chi$ is oneto-one and we can consider the induced map $G=\chi \circ \sigma \circ \chi^{-1}$ on the limit set $F$.

It immediately follows from (21) and the description of the geometric construction that there exist positive constants $C_{1}, C_{2}$ such that

$$
C_{1} \frac{\prod_{k=1}^{n} \psi\left(\sigma^{k} \omega\right)}{\prod_{k=1}^{n} \lambda_{i_{k}}} \leqq \frac{\log v\left(\Delta_{n}(x)\right)}{\log \operatorname{diam}\left(\Delta_{n}(x)\right)} \leqq C_{2} \frac{\prod_{k=1}^{n} \psi\left(\sigma^{k} \omega\right)}{\prod_{k=1}^{n} \lambda_{i_{k}}}
$$

Applying the Birkhoff Ergodic Theorem to the functions $x \rightarrow \log (\psi(x))$ and $x \rightarrow$ $\log \lambda_{i_{1}}$, where $\chi(x)=\left(i_{1} i_{2} \cdots\right)$, yields the existence of a positive number $d_{v}$ such that for $v$-almost every $x \in F$,

$$
d(x)=\lim _{n \rightarrow \infty} \frac{\log v\left(\Delta_{n}(x)\right)}{\log \operatorname{diam}\left(\Delta_{n}(x)\right)}=d_{v} .
$$

Applying Theorem 7 we obtain the following result.

Theorem 8. Let $F$ be the limit set for the symbolic geometric construction defined above. Then for any measure $v$ on the limit set $F$ such that the pullback measure $\chi^{*}(v)$ is a Gibbs measure, there exists a positive constant $d_{v}$ such that $d_{v}(x)=d_{v}$ for $v$-almost every $x \in F$.

6.2. In [ER], Eckmann and Ruelle conjectured that any hyperbolic measure (i.e., ergodic and having non-zero Lyapunov exponents almost everywhere) for a $C^{1+\alpha}$ diffeomorphism of a compact Riemannian manifold is exact dimensional, i.e. its pointwise dimension exists almost everywhere. The following theorem is a verification of the Eckmann-Ruelle Conjecture for equilibrium measures for Hölder continuous conformal expanding maps and conformal Axiom $\mathrm{A}^{\#}$ (topologically hyperbolic) homeomorphims.

\section{Theorem 9.}

(1) Let $g$ be a Hölder continuous conformal expanding map of a compact metric space $X$ (see Appendix 5). Then any equilibrium measure corresponding to a Hölder continuous function on $X$ is exact dimensional.

(2) Let $g$ be a Hölder continuous homeomorphism of a compact metric spac $X$ satisfying Axiom $A^{\#}$ (see Appendix 6). Assume that $g$ is also conformal (see 
Condition (22) in Appendix 6). Then any equilibrium measure corresponding to a Hölder continuous function on $X$ is exact dimensional.

The proof of this theorem is quite similar to the proof of Theorem 8 that is based on the fact that basic sets form a Markov partition for the induced map. The only difference is that the elements of the Markov partition for an expanding map (or topologically hyperbolic maps) are not necessarily disjoint and may intersect along their boundaries. However, the measure of the union of boundaries is zero with respect to any equilibrium measure for the expanding (or topologically hyperbolic maps). See Appendices 5 and 6 for more information on the Markov partitions.

The requirement that the map $g$ in Theorem 9 is conformal, is crucial. We present examples that illustrate the non-existence of pointwise dimension for the measure of maximal entropy of a non-conformal Hölder continuous expanding map and a Hölder continuous homeomorphism satisfying Axiom $\mathrm{A}^{\#}$. We start with the simple geometric construction presented in Example 6 and let $F$ be the corresponding limit set. Note that for this construction

$$
\underline{s} \stackrel{\text { def }}{\equiv} s_{\underline{\lambda}}=\frac{\log 2}{-\log \underline{\lambda}}, \quad \bar{s} \stackrel{\text { def }}{\equiv} s_{\bar{\lambda}}=\frac{\log 2}{-\log \bar{\lambda}} .
$$

Here the functions $\underline{\phi}(\omega)=s_{\underline{\lambda}} \log \underline{\lambda}_{i_{1}}$ and $\bar{\phi}(\omega)=s_{\bar{\lambda}}^{-} \log \bar{\lambda}_{i_{1}}$ coincide and are constant $(=\log 2)$. This implies that the Gibbs measures corresponding to these functions also coincide. Hence, $\underline{m}_{\lambda}=\bar{m}_{\lambda} \stackrel{\text { def }}{\equiv} m$; moreover $m$ is the measure of maximal entropy for the full shift $\sigma$ (see Appendix 3).

Clearly, for our construction, the coding map $\chi$ is injective and hence we can consider the map $G: F \rightarrow F$ defined by $G=\chi \circ \sigma \circ \chi^{-1}$. The map $G$ is a Hölder continuous endomorphism (but is not injective). The map $G$ is also expanding (see Appendix 5). Obviously, $m$ is an invariant measure for $G$ of maximal entropy. We describe its lower and upper pointwise dimensions. Namely, we show that for $m$-almost every $x \in F$,

$$
\underline{d}_{m}(x)=\underline{s}=\frac{\log 2}{-\log \underline{\lambda}}, \quad \bar{d}_{m}(x)=\bar{s}=\frac{\log 2}{-\log \bar{\lambda}} .
$$

The fact that $\operatorname{dim}_{H} F=\underline{s}$ immediately implies that $\underline{d}_{m}(x) \leqq \underline{s}$ for $m$-almost every $x \in F$. Otherwise there would exist a set $A$ of positive $m$-measure with $\underline{d}_{m}(x) \geqq \underline{s}+\varepsilon$ for any $x \in A$. The non-uniform mass distribution principle would then imply that $\operatorname{dim}_{H} F \geqq \operatorname{dim}_{H} A \geqq \underline{s}+\varepsilon$. Statement 2 in Theorem 4 immediately implies that $\underline{d}_{m}(x)=\frac{\log 2}{-\log \underline{\underline{\lambda}}}$.

In order to prove the corresponding result for $\bar{d}_{m}(x)$, consider $r_{k}=\bar{\lambda}^{n_{3 k+1}}$ and denote $\Delta_{k}(x)$ the unique cylinder set $\Delta_{i_{1} \cdots n_{3 k+1}}$ that contains $x \in F$. It is easy to see that $B\left(x, r_{k}\right) \cap F \subset \Delta_{k}(x) \cap F$. Since the measure $\mu$ is Gibbs the inequalities (9) imply that for all $x \in F$,

$$
m\left(B\left(x, r_{k}\right) \cap F\right) \leqq m\left(\Delta_{k}(x) \cap F\right) \leqq D_{1} \bar{\lambda}^{\bar{s} n_{3 k+1}}=D_{1} r_{k}^{\bar{s}},
$$

where $D_{1}>0$ and hence,

$$
\bar{d}_{m}(x) \geqq \limsup _{k \rightarrow \infty} \frac{\log m\left(B\left(x, r_{k}\right) \cap F\right)}{\log r_{k}} \geqq \bar{s} .
$$

We now apply Statement 2 of Proposition 4. 
6.3. As we have mentioned the map $G: F \rightarrow F$ constructed above is a Hölder continuous endomorphism but not an injective map. Consider further the set $\tilde{F}=$ $F \times F$ endowed with the metric

$$
\tilde{\rho}\left(\left(x_{1}, y_{1}\right),\left(x_{2}, y_{2}\right)\right)=\rho\left(x_{1}, x_{2}\right)+\rho\left(y_{1}, y_{2}\right), \quad x_{1}, x_{2}, y_{1}, y_{2} \in F
$$

and the coding map $\tilde{\chi}: \Sigma_{p} \rightarrow \tilde{F}$ defined by $\tilde{\chi}(x, y)=\left(\cdots i_{-1} i_{0} i_{1} \cdots\right)$, where $\Sigma_{p}$ denotes the space of two-sided sequences $\left(\cdots i_{-1} i_{0} i_{1} \cdots\right), i_{j}=1 \cdots p$ and $\chi(x)=$ $\left(\cdots i_{-2} i_{-1}\right), \chi(y)=\left(i_{0} i_{1} \cdots\right)$. Set $\tilde{G}=\tilde{\chi} \circ \sigma \circ \tilde{\chi}^{-1}$. It is easy to see that $\tilde{G}$ is a Hölder continuous homeomorphism and that for any $(x, y) \in \tilde{F}$,

$$
\pi_{1} \tilde{G}(x, y)=G(x), \quad \pi_{2} \tilde{G}^{-1}(x, y)=G(y),
$$

where $\pi_{1}, \pi_{2}$ are the projections, $\pi_{1}(x, y)=x$ and $\pi_{2}(x, y)=y$. One can also see that $\tilde{G}$ satisfies Axiom $\mathrm{A}^{\#}$ in the sense of Alekseyev and Jacobson [AJ], i.e., the map is topologically hyperbolic.

Consider the push forward measure $\tilde{m}$ of the measure $\tilde{\mu}$ on $\Sigma_{p}^{+}$defined by

$$
\tilde{\mu}\left(\Delta_{i_{k} \cdots i_{n}}\right)=\underline{\lambda}^{(n-k) \underline{s}} .
$$

By virtue of Corollary 2, $\tilde{\mu}$ is invariant under $\sigma$ and hence $\tilde{m}$ is invariant under $\tilde{G}$. It is easy to see that $\tilde{m}=m \times m$. It follows that for $\tilde{m}$-almost every $(x, y)$,

$$
\underline{d}_{\tilde{m}}(x, y)=\underline{d}_{m}(x)+\underline{d}_{m}(y)=\underline{s} \quad \bar{d}_{\tilde{m}}(x, y)=\bar{d}_{m}(x)+\bar{d}_{m}(y)=\bar{s} .
$$

It is not difficult to check that $\tilde{m}$ is the measure of maximal entropy for $\tilde{G}$. Thus we have proved the following statement.

Theorem 10. There exists a Hölder continuous Axiom $A^{\#}$ homeomorphism $G$ of a compact subset in $\mathbb{R}^{2}$ with positive topological entropy that possesses the unique measure $m$ of maximal entropy for which $\underline{d}_{m}(x) \neq \bar{d}_{m}(x)$ for $m$-almost all $x$. Moreover, $G$ is Hölder continuously conjugate to the full shift.

As we mentioned, this does not hold for smooth maps [L, PY].

6.4. We will construct an asymptotic Markov geometric construction on $[0,1]$ for which the induced map $G$ on the limit set $F$ is expanding and possesses an ergodic invariant measure with positive entropy whose pointwise dimension exists almost everywhere, and is not constant. Our approach is a simpler version than of [C].

Let $p=3$ with ratio coefficients $\lambda_{i, n}, i=1,2,3$ and $n=1,2,3, \ldots$ given by

$$
\begin{gathered}
\lambda_{1, n}=\lambda_{3, n}= \begin{cases}\alpha & \text { if } n \text { is even } \\
\beta & \text { if } n \text { is odd },\end{cases} \\
\lambda_{2, n}= \begin{cases}\gamma, & \text { if } n \text { is even } \\
\delta, & \text { if } n \text { is odd },\end{cases}
\end{gathered}
$$

where $0<\alpha \leqq \beta<\frac{1}{3}, 0<\gamma \leqq \delta<\frac{1}{3}$ and $\alpha \delta \neq \gamma \beta$. Consider the transitive matrix $A$ given by

$$
A=\left(\begin{array}{lll}
0 & 1 & 0 \\
1 & 0 & 1 \\
0 & 1 & 0
\end{array}\right)
$$

We require the following lemma. 
Lemma 5. Let $\left\{\lambda_{i, n}\right\}, i=1, \ldots, p, n=1,2, \ldots$ be sequences of numbers satisfying: $0<\lambda_{i, n}<1$, and for any $n$,

$$
\sum_{i=1}^{p} \lambda_{l, n}=\lambda<1
$$

Then there exists a sequence of affine maps $\left\{h_{i, n}\right\}$ and a geometric construction on $[0,1]$ modeled by a given symbolic dynamical system $(Q, \sigma)$ such that

(1) each basic set $\Delta_{i_{1} \cdots i_{n}}=h_{i_{1}, n} \circ \cdots \circ h_{i_{n}, n}([0,1])$;

(2) $\Delta_{i_{1} \cdots i_{n}} \cap \Delta_{j_{1} \cdots j_{n}}=\emptyset$ if $\left(i_{1} \cdots i_{n}\right) \neq\left(j_{1} \cdots j_{n}\right)$;

(3) the induced map $G=\chi \circ \sigma \circ \chi^{-1}$ on the limit set $F$ is expanding.

Proof. For each $n=1,2, \ldots$, define $h_{i, n}(x)=\lambda_{i, n} x+a_{i, n}$ and choose $a_{i, n}$ such that at each step $n$, the sets $h_{i, n}([0,1])$ are at least $\frac{\lambda}{2 p}$ apart. The result follows.

We apply Lemma 5 to the above sequence of ratio coefficients and the matrix $A$ to obtain a geometric construction on $[0,1]$ modeled by the transitive subshift of finite type $\Sigma_{A}^{+}$. It is obvious that the following limit exists:

$$
\log \lambda_{i} \stackrel{\text { def }}{\equiv} \lim _{n \rightarrow \infty} \frac{1}{n} \sum_{k=1}^{n} \log \lambda_{i, k}= \begin{cases}\frac{1}{2} \log (\beta \gamma), & \text { if } i=1,3 \\ \frac{1}{2} \log (\alpha \delta), & \text { if } i=2,\end{cases}
$$

and thus the construction is a one-dimensional asymptotic construction.

Let $\mu$ be a Gibbs measure on $\Sigma_{A}^{+}$, corresponding to a Hölder continuous function, and $v=\chi^{*} \mu$.

Consider the sets

$$
A=\left\{x \in F: \chi^{-1}(x)=\left(i_{1} i_{2} \cdots\right) \in \Sigma_{P}^{+} \text {with } i_{1}=1 \text { or } 3\right\}
$$

and

$$
B=\left\{x \in F: \chi^{-1}(x)=\left(i_{1} i_{2} \cdots\right) \in \Sigma_{P}^{+} \text {with } i_{1}=2\right\} .
$$

These sets are disjoint and comprise $\Sigma_{A}^{+}$. They are not invariant under $G$. Since $\mu$ is a Gibbs measure and the sets $\mathscr{A}$ and $\mathscr{B}$ are open, we have that $v(\mathscr{A})>0$ and $v(\mathscr{B})>0$. One can check that for every $x \in \mathscr{A}$,

$$
\lim _{n \rightarrow \infty} \frac{\log \operatorname{diam}\left(\Delta_{n}(x)\right)}{n}=\frac{1}{2} \log (\gamma \beta)
$$

and for every $x \in B$,

$$
\lim _{n \rightarrow \infty} \frac{\log \operatorname{diam}\left(\Delta_{n}(x)\right)}{n}=\frac{1}{2} \log (\alpha \delta) .
$$

Since $\chi\left(\Delta_{n}(x)\right)$ is a cylinder set, the Shannon-McMillan-Breiman Theorem implies that for $v$-almost every $x \in F$,

$$
\lim _{n \rightarrow \infty} \frac{\log v\left(\Delta_{n}(x)\right)}{n}=h_{\mu}(\sigma)>0 .
$$


Thus, by Theorem 7 , we have

$$
\begin{aligned}
\underline{d}_{v}(x) & =\bar{d}_{v}(x)=d_{v}(x)=\frac{h_{\mu}(\sigma)}{\lim _{n \rightarrow \infty}\left(\frac{\log \operatorname{diam}\left(\Delta_{n}(x)\right)}{n}\right)} \\
& =\frac{2 h_{\mu}(\sigma)}{\log (\gamma \beta)} \quad \text { for almost every } x \in A
\end{aligned}
$$

and

$$
\begin{aligned}
\underline{d}_{v}(x) & =\bar{d}_{v}(x)=d_{v}(x)=\frac{h_{\mu}(\sigma)}{\lim _{n \rightarrow \infty}\left(\frac{\log \operatorname{diam}\left(\Delta_{n}(x)\right)}{n}\right)} \\
& =\frac{2 h_{\mu}(\sigma)}{\log (\alpha \delta)} \quad \text { for almost every } x \in B .
\end{aligned}
$$

Repeating the construction in Sect. 6.3, one can prove the following statement.

Theorem 11. There exists a Hölder continuous Axiom $A^{\#}$ homeomorphism $\tilde{G}$ that possesses an invariant ergodic measure $\tilde{v}$ for which the pointwise dimension exists almost everywhere but is not essentially constant.

\section{Proofs}

Proof of Theorem 2. Let $\gamma$ be a strongly l-estimating vector. Given $r, 0<r<1$, consider the Moran cover $\mathfrak{U}_{r}=\mathfrak{U}_{r}(\gamma)$ of the set $Q$ which consists of cylinder sets $C^{(j)}=C_{i_{1} \cdots l_{n\left(\omega_{j}\right)}}, j=1, \ldots, N$. Let $\Delta_{i_{1} \cdots l_{n\left(x_{j}\right)}}=\chi\left(C_{\left.i_{1} \cdots i_{n\left(\omega_{j}\right)}\right)}\right)$, where $x_{j}=\chi\left(\omega_{j}\right)$.

By the definition of a Gibbs measure (see Appendix 3 ) there exist positive constants $D_{1}$ and $D_{2}$ such that for $j=1, \ldots, N$,

$$
D_{1} \leqq \frac{m_{\gamma}\left(\Delta_{i_{1} \cdots i_{n\left(x_{j}\right)}}\right)}{\prod_{k=1}^{n\left(x_{j}\right)} \gamma_{i_{k}}^{s_{\gamma}}} \leqq D_{2},
$$

where $m_{\gamma}$ is the push forward of the Gibbs measure $\mu_{\gamma}$ corresponding to the function $\phi\left(i_{1} i_{2} \cdots\right)=s_{\gamma} \log \gamma_{t_{1}}$ on $Q$. Since the vector $\gamma$ is strongly l-estimating, by (9), we have

$$
\begin{aligned}
m_{\gamma}(B(x, r)) & \leqq \sum_{j=1}^{N(x, r)} m_{\gamma}\left(\Delta^{(j)}\right) \leqq \sum_{j=1}^{N(x, r)} D_{2} \prod_{k=1}^{n\left(x_{j}\right)} \gamma_{i_{k}}^{s_{\gamma}} \\
& \leqq L_{4} \sum_{j=1}^{N(x, r)} \prod_{k=1}^{n\left(x_{j}\right)+1} \gamma_{i_{k}}^{s_{i}} \leqq L_{4} N(x, r) r^{s_{\gamma}} \leqq L_{5} r^{s_{;}},
\end{aligned}
$$

where $L_{4}, L_{5}>0$ are constants. Hence the measure $m_{\gamma}$ satisfies the uniform mass distribution principle. This proves statement (1). Moreover, (10) implies that $s_{\gamma} \leqq \underline{d}_{m}(x)$ for every $x \in F$. It follows that $s_{\gamma} \leqq \operatorname{dim}_{H} F$ and $m_{H}\left(s_{\gamma}, F\right)>0$.

We now prove that $m_{\gamma}(Z) \leqq m_{H}(s, Z)$ for any Borel subset $Z \subset F$. Given $\delta>0$, there exists $\varepsilon>0$ and a covering $\mathfrak{U}=\left\{U^{(k)}\right\}$ of $Z$ by open sets $U^{(k)}$ with 
$\operatorname{diam} U^{(k)} \leqq \varepsilon$ satisfying

$$
\sum_{U^{(k)} \in \mathfrak{U}}\left(\operatorname{diam} U^{(k)}\right)^{s} \leqq m_{H}(s, Z)+\delta
$$

By (10) it follows that

$$
m_{\gamma}(Z) \leqq \sum_{U^{(k)} \in \mathfrak{l}} m_{\gamma}\left(U^{(k)}\right) \leqq L_{5} \sum_{U^{(k)} \in \mathfrak{U}}\left(\operatorname{diam} U^{(k)}\right)^{s} \leqq L_{5} m_{H}(s, Z)+L_{5} \delta .
$$

Since $\delta$ is chosen arbitrarily this implies that $m_{\gamma}(Z) \leqq m_{H}(s, Z)$.

Proof of Proposition 2. We begin with the following general lemma:

Lemma 6. Let $A=(A(i, j))$ be a transitive $(p \times p)$ matrix of $0 s$ and $1 s$, and consider the subshift of finite type defined by $A$. Let $f: \Sigma_{A}^{+} \rightarrow \mathbb{R}$ be a continuous function that depends only on the first coordinate. Let $F$ denote the $(p \times p)$ diagonal matrix $\operatorname{diag}\left(e^{f(1)}, e^{f(2)}, \ldots, e^{f(p)}\right)$. Then $P_{A}(\log f(\omega))=P_{A}\left(\log f\left(\omega_{1}\right)\right)=$ $\log r$, where $r$ denotes the spectral radius of the $(p \times p)$ matrix $A^{*} F$, where $A^{*}$ denotes the transpose of $A$.

Proof. In the proof, we exploit the fact that the exponential of the pressure is the maximal eigenvalue of the transfer operator. Let $\phi: \Sigma_{A}^{+} \rightarrow \mathbb{R}$ be a continuous function. Then the transfer operator

$$
\left(L_{f} \phi\right)(x) \stackrel{\text { def }}{\equiv} \sum_{k} \exp (f(k x)) \phi(k x) A\left(k, x_{1}\right)=\sum_{k} \exp (f(k x)) \phi(k x) A\left(k, x_{1}\right),
$$

where $x=\left(x_{1} x_{2} \cdots\right)$. The eigenvalue equation for $L_{f}$ is

$$
\sum_{k} \exp (f(k)) h(k) A(k, j)=\eta h(j) .
$$

According to [PP, p. 24 (note normalization of the transfer operator)], the largest eigenvalue of $L_{f}$ is $\eta=\exp (P(f))$. Hence $\exp (P(f))$ is the spectral radius of the matrix $A^{*} F$.

Proposition 2 follows by applying Lemma 6 to the function $f(\omega)=f\left(\omega_{1}\right)=$ $t \log \alpha_{()_{1}}$.

Proof of Theorem 4. By the definition of a Gibbs measure, there exist constants $D_{1}, D_{2}>0$ such that for any basic set $\Delta_{l_{1} \cdots i_{n}}$,

$$
D_{1} \leqq \frac{m_{\lambda}\left(\Delta_{i_{1} \cdots i_{n}}\right)}{\prod_{k=1}^{n} \lambda_{l_{k}}^{s_{\prime}}} \leqq D_{2} .
$$

Fix $0<r<1$. For each $x \in F$, choose a point $\omega=\left(i_{1} i_{2} \cdots\right) \in Q$ such that $\chi(\omega)=x$. Define $1 \leqq i_{n(\omega)} \leqq p$ such that $\lambda_{i_{1}} \lambda_{i_{2}} \cdots \lambda_{i_{n(\omega)}}>r$ and $\lambda_{l_{1}} \lambda_{l_{2}} \cdots \lambda_{i_{n(\omega)}} \lambda_{i_{n(\omega)+1}} \leqq r$. It follows that $\Delta_{l_{1} \cdots l_{n(\omega)+1}}=\chi\left(C_{i_{1} \cdots i_{n(o)+1}}\right) \subset B\left(x, 2 C_{2} r\right)$, where $B(x, r)$ denotes the ball 
of radius $r$ around the point $x$. Hence, for all $x \in F$,

$$
m_{\lambda}\left(B\left(x, 2 C_{2} r\right)\right) \geqq m_{\lambda}\left(\Delta_{i_{1} \cdots i_{n(\omega)+1}}\right) \geqq D_{1} \prod_{k=1}^{n(\omega)+1} \lambda_{i_{k}}^{s_{1}} \geqq L_{1} r^{s,},
$$

where $L_{1}>0$ is a constant. It follows by (11) that for all $x \in F$,

$$
\bar{d}_{m_{\lambda}}(x)=\limsup _{r \rightarrow 0} \frac{\log m_{\lambda}(B(x, r))}{\log r} \leqq s_{\lambda} .
$$

We now prove that $m_{H}(s, \cdot) \leqq$ const $m_{\lambda}(\cdot)$. Let $Z \subset F$ be a closed subset. Given $\delta>0$, there exists $\varepsilon>0$ such that for any covering $\mathfrak{U}=\left\{U^{(k)}\right\}$ of $Z$ by open sets $U^{(k)}$ with $\operatorname{diam} U^{(k)} \leqq \varepsilon$, we have

$$
m_{H}(s, Z) \leqq \sum_{U^{(k)} \in \mathfrak{U}}\left(\operatorname{diam} U^{(k)}\right)^{s}+\delta
$$

There exists a covering $\mathfrak{U}$ of $Z$ by basic sets $\Delta^{(k)}=\Delta_{i_{1} \cdots i_{n(k)}}$ satisfying diam $\Delta^{(k)} \leqq \varepsilon$ and

$$
\sum_{\Delta^{(k)} \in \mathfrak{U}} m_{\lambda}\left(\Delta^{(k)}\right) \leqq m_{\lambda}(Z)+\delta .
$$

Since $\lambda$ is a $\mathrm{u}$-estimating vector, it follows from (2) that

$$
\begin{aligned}
m_{H}(s, Z) & \leqq \sum_{\Delta^{(k)} \in \mathfrak{U}}\left(\operatorname{diam} \Delta^{(k)}\right)^{s}+\delta \leqq C_{2} \sum_{\Delta^{(k)} \in \mathfrak{U}} \prod_{j=1}^{n(k)} \lambda_{i_{j}}^{s}+\delta \\
& \leqq C_{2} D_{2} \sum_{\Delta^{(k)} \in \mathfrak{U}} m_{\lambda}\left(\Delta^{(k)}\right)+\delta \leqq C_{2} D_{2} m_{\lambda}(Z)+\left(C_{2} D_{2}+1\right) \delta .
\end{aligned}
$$

Since $\delta$ is chosen arbitrarily this implies the desired result.

Proof of Theorem 1. Given numbers $0<\lambda_{1}, \lambda_{2}, \ldots, \lambda_{p}<1$, let $\mu_{\lambda}$ be an equilibrium measure on $Q$ corresponding to the function $s_{\lambda} \log \lambda_{i_{1}}$. By definition,

$$
h_{\mu ;}(\sigma \mid Q)+s_{\lambda} \int_{Q} \log \lambda_{i_{1}} d \mu_{\lambda_{2}}=0
$$

where $h_{\mu}(\sigma \mid Q) \stackrel{\text { def }}{\equiv} h$ is the Kolmogorov-Sinai entropy. Let us first assume that $\mu_{\lambda}$ is ergodic. For fixed $\varepsilon>0$, it follows from the Shannon-McMillan-Breiman theorem that for $\mu_{\lambda}$-almost every $\omega \in Q$ one can find $N_{1}(\omega)>0$ such that for any $n \geqq$ $N_{1}(\omega)$,

$$
\exp (-(h+\varepsilon) n) \leqq \mu_{\lambda}\left(C_{i_{1} \cdots i_{n}(\omega)}\right) \leqq \exp (-(h-\varepsilon) n),
$$

where $C_{i_{1} \cdots i_{n}(\omega)}$ is the cylinder set containing $\omega$. It follows from the Birkhoff Ergodic

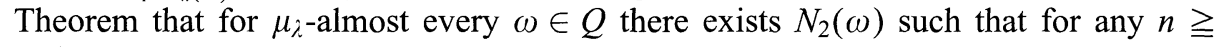
$N_{2}(\omega)$,

$$
\frac{1}{n} \log \prod_{j=1}^{n} \lambda_{i_{j}}^{s}-\varepsilon \leqq s \int_{Q} \log \lambda_{i_{1}} d \mu_{\lambda} \leqq \frac{1}{n} \log \prod_{j=1}^{n} \lambda_{i,}^{s}+\varepsilon
$$


Combining (12), (13), and (14) we have that for $\mu_{\lambda}$-almost every $\omega \in Q$ and $n$ sufficiently large,

$$
\prod_{j=1}^{n} \lambda_{i_{j}}^{s+\alpha} \leqq \prod_{j=1}^{n} \lambda_{i_{j}}^{s} \exp (-2 \varepsilon n) \leqq \mu_{\lambda}\left(C_{l_{1} \cdots i_{n}(\omega)}\right) \leqq \prod_{j=1}^{n} \lambda_{i_{j}}^{s} \exp (2 \varepsilon n) \leqq \prod_{j=1}^{n} \lambda_{i_{j}}^{s-\alpha},
$$

where $\alpha=\frac{2 \varepsilon}{\operatorname{mn}\left(\log \frac{1}{1}, 1 \leqq j \leqq p\right)}>0$. This implies that for $\mu_{\lambda}$-almost every $\omega \in Q$ and any $n \geqq \max \left\{N_{1}(\omega), N_{2}(\omega)\right\}$,

$$
\prod_{j=1}^{n} \lambda_{i_{j}}^{s+\alpha} \leqq \mu_{\lambda}\left(C_{i_{1} \cdots i_{n}(\omega)}\right) \leqq \prod_{j=1}^{n} \lambda_{i_{j}}^{s-\alpha}
$$

If $\mu_{\lambda}$ is not ergodic, then (15) is still valid which can be shown by decomposing $\mu$ into its ergodic components.

Given $l>0$ denote $Q_{l}=\left\{\omega \in Q: N_{1}(\omega) \leqq l\right.$ and $\left.N_{2}(\omega) \leqq l\right\}$. It is easy to see that $Q_{l} \subset Q_{l+1}$ and $Q=\bigcup_{l=1}^{\infty} Q_{l}(\bmod 0)$. Thus there exists $l_{0}>0$ such that $\mu\left(Q_{l}\right)>0$ if $l \geqq l_{0}$. Let $x \in \chi\left(Q_{l}\right), l \geqq l_{0}, 0<r<1$.

Repeating the arguments in the proof of Theorem 2 with $Q$ replaced by $Q_{l}$ and applying (15) to the numbers $\gamma_{1}, \ldots, \gamma_{p}$ and measure $\mu_{\gamma}$, one obtains that $m_{\gamma}(B(x, r) \cap$ $\left.\chi\left(Q_{l}\right)\right) \leqq K r^{s_{\gamma}-\alpha}$, where $K=K(l)>0$ is a constant and $\left.x \in \chi^{-1}\left(Q_{l}\right)\right)$ is any point. This implies that for any $l>0$ and $x \in \chi^{-1}\left(Q_{l}\right)$,

$$
\underline{d}_{m_{\gamma}}^{l}(x) \stackrel{\text { def }}{\equiv} \liminf _{r \rightarrow 0} \frac{\log m_{\gamma}\left(B(x, r) \cap \chi\left(Q_{l}\right)\right)}{\log r} \geqq s_{\gamma}-\alpha .
$$

It follows that for all sufficiently large $l \in \mathbb{N}$, $\operatorname{dim}_{H} Q_{l} \geqq s_{\gamma}-\alpha$. This implies that $\operatorname{dim}_{H} F \geqq s_{\gamma}-\alpha$. Since $\alpha$ can be arbitrarily small, this gives the desired result.

Proof of Theorem 3. Fix $0<r<1$. For any $\omega=\left(i_{1} i_{2} \cdots\right) \in Q$, define the unique $n(\omega)$ such that $\lambda_{i_{1}} \lambda_{i_{2}} \cdots \lambda_{i_{n(\omega)}}>r$ and $\lambda_{i_{1}} \lambda_{l_{2}} \cdots \lambda_{i_{n(\omega)+1}} \leqq r$. It is easy to see that $n(\omega) \rightarrow \infty$ as $r \rightarrow 0$ uniformly in $\omega$. Fix $l>0$ for which $\mu_{\lambda}\left(Q_{l}\right)>0$, where $Q_{l}$ are the sets constructed in the proof of Theorem 1. One can now choose $r=r(l)>0$ sufficiently small such that $n(\omega)$ becomes large enough to satisfy (15) for any $\omega \in Q_{l}$. Repeating the arguments in the proof of Theorem 4 with $Q$ replaced by $Q_{l}$, and applying $(15)$ to the numbers $\left(\lambda_{1}, \ldots, \lambda_{p}\right)$ and the measure $\mu_{\lambda}$, one can show that for any $x \in \chi\left(Q_{l}\right)$ and any $r>0$ sufficiently small,

$$
m_{\lambda}(B(x, r)) \geqq K r^{s,+\alpha},
$$

where $K=K(l)>0$ is a constant. This implies that $\bar{d}_{m_{\lambda}}(x) \leqq s_{\lambda}+\alpha$ and hence, $\bar{d}_{m,}(x) \leqq s_{i}+\alpha$ for any $x \in F$. This completes the proof of statement (2).

We now show that $\overline{\operatorname{dim}}_{B} F \leqq s_{\lambda}$. The arguments in this proof are essentially due to Bowen and were pointed out to us by Ledrappier.

It is sufficient to prove that $P\left(\overline{\operatorname{dim}}_{B} F \log \lambda_{i_{1}}\right) \geqq 0$, since the map $t \rightarrow P\left(t \log \lambda_{i_{1}}\right)$ is a decreasing function [Bol].

Given $\delta>0$, it follows from the definition of $\overline{\operatorname{dim}}_{B} F$ (see Appendix 1) that there exists $\varepsilon>0$ such that $N_{\varepsilon}(F) \geqq \varepsilon^{\delta-\overline{\operatorname{dim}}_{B} F}$. Consider the Moran cover $\left\{C^{(j)}\right\}$ $=\left\{C_{\left.i_{1}, \ldots, i_{n(\omega,)}\right)}\right\}, j=1, \ldots, N^{\varepsilon}(F)$. Note that this cover need not be optimal, i.e., $N^{\varepsilon}(F)$ 
$\geqq N_{\varepsilon}(F)$. There clearly exists $A=A(\varepsilon)>0$ such that for $j=1, \ldots, N^{\varepsilon}(F)$,

$$
\frac{\varepsilon}{A} \leqq \prod_{k=1}^{n\left(\omega_{j}\right)} \lambda_{i_{k}} \leqq \varepsilon,
$$

and hence

$$
C_{1} \log \left(\frac{1}{\varepsilon}\right) \leqq n\left(\omega_{J}\right) \leqq C_{2} \log \left(\frac{A}{\varepsilon}\right)
$$

where $C_{1}=\frac{1}{\log \left(\frac{1}{\gamma_{\max }}\right)}$ and $C_{2}=\frac{1}{\log \left(\frac{1}{i_{\text {min }}}\right)}$. This implies that $n\left(\omega_{j}\right)$ can take on at most $C_{2} \log \left(\frac{A}{\varepsilon}\right)-C_{1} \log \left(\frac{1}{\varepsilon}\right)$ possible values.

We now think of having $N_{\varepsilon}(F)$ balls and $C_{2} \log \left(\frac{A}{\varepsilon}\right)-C_{1} \log \left(\frac{1}{\varepsilon}\right)$ baskets. Then for $N^{\varepsilon}(F) \geqq C_{2} \log \left(\frac{A}{\varepsilon}\right)-C_{1} \log \left(\frac{1}{\varepsilon}\right)$, there exists a basket containing at least $\frac{N^{\varepsilon}(F)}{C_{2} \log \left(\frac{A}{\varepsilon}\right)-C_{1} \log \left(\frac{1}{\varepsilon}\right)}$ balls. This implies that there exists a positive integer $\alpha$ with $C_{1} \log \left(\frac{1}{\varepsilon}\right) \leqq \alpha \leqq C_{2} \log \left(\frac{A}{\varepsilon}\right)$ such that for $\varepsilon$ sufficiently small,

$$
\begin{gathered}
\#\left\{\omega_{j} \text { such that } n\left(\omega_{J}\right)=\alpha\right\} \geqq \frac{N^{\varepsilon}(F)}{C_{2} \log \left(\frac{A}{\varepsilon}\right)-C_{1} \log \left(\frac{1}{\varepsilon}\right)} \\
\geqq \frac{N_{\varepsilon}(F)}{C_{2} \log \left(\frac{A}{\varepsilon}\right)-C_{1} \log \left(\frac{1}{\varepsilon}\right)} \geqq \frac{\varepsilon^{\delta-\overline{\operatorname{dim}}_{B} F}}{C_{3} \log \left(\frac{1}{\varepsilon}\right)} \geqq \varepsilon^{2 \delta-\overline{\operatorname{dim}}_{B} F} .
\end{gathered}
$$

Let $\phi(\omega)=\left(\overline{\operatorname{dim}}_{B} F-2 \delta\right) \log \lambda_{i_{1}}$, where $\omega=\left(i_{1} i_{2} \cdots\right)$. Then

$$
\left(S_{n} \phi\right)(\omega)=\sum_{k=1}^{n} \phi\left(\sigma^{k} \omega\right)=\left(\overline{\operatorname{dim}}_{B} F-2 \delta\right) \log \prod_{k=1}^{n} \lambda_{i_{k}},
$$

and hence $\exp \left(S_{n} \phi\right)(\omega)=\left(\prod_{k=1}^{n} \lambda_{i_{k}}\right)^{\overline{\operatorname{dim}}_{B} F-2 \delta}$. It follows that

$$
\begin{aligned}
& P_{\alpha}\left(\left(\overline{\operatorname{dim}}_{B} F-2 \delta\right) \log \lambda_{1_{1}}\right) \stackrel{\text { def }}{\equiv} \frac{1}{\alpha} \log \sum_{\substack{\left.i_{1} \cdots i_{\alpha}\right) \\
\text { admissible }}} \inf _{\omega \in C_{i_{1} \cdot i_{\alpha}}}\left(\prod_{k=1}^{n} \lambda_{i_{k}}\right)^{\overline{\operatorname{dim}}_{B} F-2 \delta} \\
& \geqq \frac{1}{\alpha} \log \sum_{\substack{\left(i_{1} \cdots i_{\alpha}\right) \\
\text { in covering }\left\{\Delta^{(\jmath)}\right\}}}\left(\frac{A}{\varepsilon}\right)^{\overline{\operatorname{dim}}_{B} F-2 \delta} \\
& \geqq A^{\overline{\operatorname{dim}}_{B} F-2 \delta} \frac{1}{\alpha} \log \left(\varepsilon^{\overline{\operatorname{dim}}_{B} F-2 \delta} \cdot \varepsilon^{2 \delta-\overline{\operatorname{dim}}_{B} F}\right) \geqq 0
\end{aligned}
$$

Hence $P\left(\left(\overline{\operatorname{dim}}_{B} F-2 \delta\right) \log \lambda_{i_{1}}\right)=\lim _{\alpha \rightarrow \infty} P_{\alpha}\left(\left(\overline{\operatorname{dim}}_{B} F-2 \delta\right) \log \lambda_{i_{1}}\right)=0$.

Proof of Proposition 1. Let $\mu_{\lambda}$ denote an equilibrium measure for $s_{\lambda} \log \lambda_{i_{1}}$. It immediately follows from the variational principle that

$$
s_{\lambda}=\frac{h_{\mu_{i}}(\sigma \mid Q)}{-\int \log \lambda_{i_{1}} d \mu_{\lambda}} \leqq \frac{h(\sigma \mid Q)}{-\log \max \lambda_{k}} .
$$

The case of equality is obvious.

If $\lambda_{i}=\lambda$ for $i=1, \ldots, p$, then this immediately implies that $s_{\lambda}=\frac{h(\sigma \mid Q)}{-\log \lambda}$. This proves the desired results. 


\section{Proof of Proposition 7.}

1) The first inequality in (4) implies that for any $\varepsilon>0$ and $\mu_{\lambda}$-almost every $\omega \in Q$, $\lambda=\left(\lambda_{1}, \ldots, \lambda_{p}\right)$, there exists $N_{3}(\omega)=N_{3}(\omega, \varepsilon)$ such that for any $n \geqq N_{3}(\omega)$,

$$
\left|\frac{1}{n} \sum_{j=1}^{n} \underline{a}_{i, j}\right| \leqq \varepsilon .
$$

The inequality (16) is equivalent to

$$
\prod_{J=1}^{n} \lambda_{i_{j}} \exp (-\varepsilon n) \leqq \prod_{j=1}^{n} \underline{\lambda}_{i_{j}, j} \leqq \prod_{j=1}^{n} \lambda_{i_{j}} \exp (\varepsilon n) .
$$

It is sufficient to consider only the case when $\mu_{\lambda}$ is ergodic with respect to $\sigma$. Then for $\mu_{i}$-almost every $\omega=\left(i_{1} i_{2} \cdots\right) \in Q$, the following limit exists:

$$
\lim _{n \rightarrow \infty} \sum_{j=1}^{n} \log \lambda_{i_{j}}=\int_{Q} \log \lambda_{\omega_{1}} d \mu_{\lambda}(\omega) \equiv a<0 .
$$

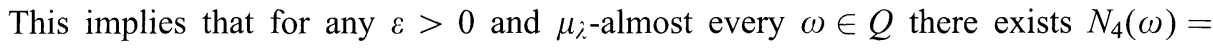
$N_{4}(\omega, \varepsilon)$ such that for any $n \geqq N_{4}(\omega)$,

$$
\left|\frac{1}{n} \sum_{j=1}^{n} \log \lambda_{i_{J}}-a\right| \leqq \varepsilon .
$$

Given $l>0$ denote

$$
Q_{l}=\left\{\omega \in Q: N_{i}(\omega) \leqq l, i=1,2,3,4\right\},
$$

where $N_{1}(\omega), N_{2}(\omega)$ are the two functions constructed in the proof of Theorem 1 (see (15)). It is easy to see that $Q_{l} \subset Q_{l+1}$ and $Q=\bigcup_{l=1}^{\infty} Q_{l} \bmod (0)$. Thus there exists $l_{0}>0$ such that $\mu_{\lambda}\left(Q_{l}\right)>0$ if $l \geqq l_{0}$.

Consider $\omega=\left(i_{1} i_{2} \cdots\right) \in Q_{l}, l \geqq l_{0}$ and $0<r<1$. Let $n(\omega)$ denote the unique positive integer such that $\lambda_{i_{1}} \lambda_{i_{2}} \cdots \lambda_{l_{l(\omega)}}>r$ and $\lambda_{i_{1}} \lambda_{i_{2}} \cdots \lambda_{l_{\text {l(o) }}+1} \leqq r$. Obviously, $n(\omega) \rightarrow \infty$ as $r \rightarrow 0$ uniformly in $\omega$. Hence we can assume $r=r(l)$ is so small that $n(\omega) \geqq \max \left\{N_{3}(\omega), N_{4}(\omega)\right\}$. Applying (18) to $n=n(\omega)$ we have

$$
n(\omega) \leqq \frac{\log r}{(a+\varepsilon)} .
$$

2) Consider the cylinder set $C_{l_{1} \cdots l_{(\omega)}}$. We have that $\omega \in C_{i_{1} \cdots i_{n(o)}}$ and if $\omega^{\prime} \in C_{i_{1} \cdots i_{n(\omega)}} \cap Q_{l}$ and $n\left(\omega^{\prime}\right) \leqq n(\omega)$ then

$$
C_{i_{1} l_{2} \cdots i_{n\left(\omega^{\prime}\right)}} \subset C_{i_{1 l_{2}} \cdots l_{n(\omega)}} .
$$

Let $C(\omega)$ be the largest cylinder set containing $\omega$ with the property that for every $\omega^{\prime} \in C(\omega) \cap Q_{l}$ we have $C_{i_{1} \cdots i_{n\left(\omega^{\prime}\right)}} \cap Q_{l} \subset C(\omega) \cap Q_{l}$ and there exists $\tilde{\omega} \in C(\omega)$ such that $C(\omega)=C_{l_{1} \cdots i_{n(\omega)} \text {. }}$. It is easy to see that the sets $C(\omega) \cap Q_{l}$ corresponding to different $\omega \in Q_{l}$ either coincide or are disjoint. We denote these sets by $C^{(\prime)}, j=1, \ldots, N$. There exist points $\omega_{\text {, }}$ such that $C^{(j)}=C_{i_{1} \cdots i_{(\omega, j)}}$. For any 
$j=1, \ldots, N$ we have

$$
l^{-1} \prod_{k=1}^{n\left(\omega_{J}\right)} \lambda_{i_{k}}^{s_{\prime}} \leqq \mu_{\lambda}\left(C^{(j)}\right) \leqq l \prod_{k=1}^{n\left(\omega_{J}\right)} \lambda_{i_{k}}^{s_{i}} .
$$

Consider a point $x=\chi(\omega) \in F$ with $\omega \in Q_{l}$. We estimate the number $N(x, r)$ of sets $C^{(j)}$ that intersect the set $B(x, r) \cap \chi^{-1}\left(Q_{l}\right)$ for sufficiently small $r$. It follows from (17) and (18) that

$$
\begin{aligned}
N(x, r) & \leqq \frac{\operatorname{vol}\left(B\left(x, r+\max _{1 \leqq j \leqq N}\left(\operatorname{diam}\left(\chi\left(C^{(j)}\right)\right)\right)\right)\right)}{\min _{1 \leqq j \leqq N} \operatorname{vol}\left(\chi^{-1}\left(C^{(j)} \cap Q_{l}\right)\right)} \\
& \leqq \frac{\max _{1 \leqq j \leqq N} \operatorname{vol}\left(B\left(x, r+\frac{r}{\lambda_{\min }} \exp \left(\varepsilon n\left(w_{j}\right)\right)\right)\right)}{\min _{1 \leqq j \leqq N} \operatorname{vol} B\left(x, C_{1} r^{\frac{a}{a+\varepsilon}}\right)} \\
& \leqq C_{2} \frac{\left(r\left(1+\frac{1}{\lambda_{\min }} r^{\frac{\varepsilon}{a+\varepsilon}}\right)\right)^{d}}{\left(r^{\frac{x}{a+\varepsilon}}\right)^{d}} \leqq C_{3} r^{-b \varepsilon},
\end{aligned}
$$

where $C_{1}, C_{2}, C_{3}, b$ are positive constants. This implies that the construction is conditionally regular.

3) The proof follows from 2).

The proof of Proposition 8 is a slight modification of the proof of Proposition 7.

Proof of Proposition 3. Obviously Moran-like constructions are bounded. The regularity of these constructions immediately follows from Condition 3 since the number of disjoint balls of a given radius $r$ which intersect a given ball of radius constant $\times r$, is uniformly bounded, independently of $r$.

The second statement follows from Theorem 5.

Proof of Lemma 1. We will prove Statement (1). The proof of Statement (2) is similar. Applying the Birkhoff Ergodic Theorem for the stationary ergodic process to the functions $\underline{f}(\vec{\lambda})=\left(\log \underline{\lambda}_{1,1}, \ldots, \log \underline{\lambda}_{p, 1}\right)$ and $\bar{f}(\vec{\lambda})=\left(\log \bar{\lambda}_{1,1}, \ldots, \log \bar{\lambda}_{p, 1}\right)$ we obtain

$$
\frac{1}{n} \sum_{k=1}^{n} \log \underline{\lambda}_{i, k} \rightarrow \log \underline{\lambda}_{l}
$$

for $v$ almost every $\vec{\lambda}$, where we define $\log \underline{\lambda}_{i}$ as the limiting value. We need to show that for $\underline{\mu}$ almost every $\omega=\left(i_{1} i_{2} \cdots\right) \in Q$,

$$
\lim _{N \rightarrow \infty} \frac{1}{N} \sum_{k=1}^{N} \log \left(\frac{\underline{\lambda}_{i_{k}, k}}{\underline{\lambda}_{i_{k}}}\right)=0 .
$$

We break up the sum

$$
\begin{aligned}
& \frac{1}{N} \sum_{k=1}^{N} \log \left(\frac{\underline{\lambda}_{i_{k}, k}}{\underline{\lambda}_{i_{k}}}\right)=\frac{1}{N} \sum_{j=1}^{p} \sum_{\substack{i_{k}=j \\
1 \leqq k \leqq N}} \log \left(\frac{\underline{\lambda}_{j, k}}{\underline{\lambda}_{j}}\right) \\
& =\sum_{j=1}^{p} \frac{\#\left\{i_{k}=j\right\}}{N}\left(\frac{1}{\#\left\{i_{k}=j\right\}} \sum_{\substack{i_{k}=j \\
1 \leqq k \leqq N}} \log \left(\frac{\underline{\lambda}_{j, k}}{\underline{\lambda}_{j}}\right)\right) .
\end{aligned}
$$


We wish to show that for $\mu$-almost every $\omega$, each of the above terms in parentheses tends to zero as $N$ tends to infinity. This immediately follows from the following generalization of the Birkhoff Ergodic Theorem for return times to a set.

Theorem [BFKO]. Let $(X, \mathfrak{B}, v, T)$ and $(Y, \mathfrak{C}, \mu, S)$ be two mixing measure theoretic dynamical systems. Let $A \subset Y$ be of positive measure, $\mu(A)>0$. For every $y \in Y$ let $A_{y}$ denote the return time sequence $\left\{n \in \mathbb{N} \mid S^{n} y \in A\right\}$. Then for $\mu$-almost every $y \in Y$ and for $f \in L^{1}(X)$,

$$
\lim _{N \rightarrow \infty} \frac{1}{N} \sum_{\substack{1 \leq k \leq N \\ k \in \bar{A}_{v}}} T^{k} f(x)=\int_{X} f d v
$$

for v-almost every $x \in X$.

We apply this theorem for $T$ the ergodic stationary process in the definition of random symbolic geometric construction, $S=\sigma: Q \rightarrow Q$ the shift map with invariant Gibbs measure $\mu$, and $A=C_{j}=\left\{\omega \in Q \mid \omega_{1}=j\right\}$. Clearly, the set $\left\{1 \leqq k \leqq N, i_{k}=j\right\}=\left\{1 \leqq k \leqq N, \sigma^{k} \omega \in C_{j}\right\}$.

Proof of Theorem 7. Given $x \in F$ let $\omega=\left(i_{1} i_{2} \cdots\right) \in Q$ be the unique point such that $x=\chi(\omega)$. Fix $r>0$ and choose $n_{r}(\omega)$ such that $C_{2} \prod_{k=1}^{n,(\omega)} \lambda_{i_{k}}<r$ and $C_{2} \prod_{k=1}^{n,(\omega)-1} \lambda_{i_{k}} \geqq r$. Since $\omega \in C_{i_{1} i_{2} \cdots i_{n}}$, where $n=n_{r}(\omega)$ we have $\Delta_{i_{1} i_{2} \cdots i_{n}} \subset B(x, r)$, where $\Delta_{i_{1} l_{2} \cdots i_{n}}=\chi\left(C_{i_{1} i_{2} \cdots i_{n}}\right)$. This implies

$$
\frac{\log v(B(x, r))}{\log r} \leqq \frac{\log v\left(\Delta_{i_{1} i_{2} \cdots i_{n}}(x)\right)}{\log r} .
$$

Since this inequality holds for all $\omega \in Q$ with $\chi(\omega)=x$ we obtain

$$
\frac{\log v(B(x, r))}{\log r} \leqq \frac{\log v\left(\Delta_{l_{1} i_{2} \cdots i_{n}}(x)\right)}{\log r}
$$

We also have

$$
r \leqq C_{2} \prod_{k=1}^{n-1} \lambda_{l_{k}}=C_{2} \prod_{k=1}^{n} \lambda_{i_{k}} \frac{1}{\lambda_{i_{n}}} \leqq \frac{C_{2}}{C_{1} \lambda_{i_{n}}} \operatorname{diam} \Delta_{l_{1} i_{2} \cdots i_{n}} \leqq C_{3} \operatorname{diam} \Delta_{i_{1} i_{2} \cdots l_{n}},
$$

where $n=n_{r}(\omega)$ and $C_{3}>0$ is a constant.

It follows that

$$
\begin{aligned}
\bar{d}_{v}(x) & =\limsup _{r \rightarrow 0} \frac{\log v(B(x, r))}{\log r} \leqq \limsup _{r \rightarrow 0} \frac{\log v\left(\Delta_{i_{1} i_{2} \cdots i_{n_{r}(\omega)}}\right)}{\log \operatorname{diam} \Delta_{i_{1} i_{2} \cdots i_{n_{r}(\omega)}}} \\
& \leqq \limsup _{r \rightarrow 0} \frac{\log v\left(\Delta_{i_{1} i_{2} \cdots i_{n}}(x)\right)}{\log \operatorname{diam} \Delta_{i_{1} i_{2} \cdots i_{n}(x)}}=\bar{d}(x) .
\end{aligned}
$$

We now prove the second estimate in Statement 1 . Given $k \in \mathbb{N}$, define $F_{C, \alpha}=$ $\left\{x \in F: v\left(\Delta_{n}(x)\right) \leqq C\left(\operatorname{diam} \Delta_{n}(x)\right)^{\alpha}\right.$ for all $\left.n \geqq 0\right\}$. If $v\left(F_{C, \alpha}\right)>0$, then by the general density theorem [Fe], $v$-almost every point in $F_{C, \alpha}$ is a point of density. Fix 
a set $F_{C, \alpha}$ having positive measure and let $x$ be a point of density. It follows that there exists $R=R(x)$ such that if $r \leqq R$ then $v(B(x, r)) \leqq 2 v\left(B(x, r) \cap F_{C, \alpha}\right)$.

Consider the set $A=\chi^{-1}\left(F_{C, \alpha}\right)$. For $r<R(x)$ consider the Moran cover $\mathfrak{U}_{r, A}(\lambda)$ of $F_{C, \alpha}$. This cover consists of $M$ disjoint cylinder sets $C^{(j)}=C_{\left.i_{1} \cdots i_{n(\omega)}\right)}$, where $\omega_{j} \in A$ and $M$ is the Moran multiplicity factor. Moreover, $\prod_{k=1}^{n\left(x_{j}\right)} \lambda_{i_{k}} \leqq r$, and $B(x, r) \cap F_{k} \subset \bigcup_{j=1}^{M}\left(\Delta^{(j)} \cap F_{k}\right)$, where $\Delta^{(j)}=\chi\left(C^{\jmath}\right)$. If follows that

$$
v(B(x, r)) \leqq 2 v\left(B(x, r) \cap F_{C, \alpha}\right) \leqq 2 \sum_{j=1}^{M} v\left(\Delta^{(j)}\right) \leqq 2 C \sum_{j=1}^{M} \operatorname{diam}\left(\Delta^{(j)}\right)^{\alpha} \leqq 2 M C r^{\alpha} .
$$

Hence $\underline{d}_{v}(x) \geqq \alpha$. On the other hand, it is clear that $\underline{d}(x) \leqq \alpha$ for every $x \in F_{C, \alpha}$. It follows that $\underline{d}(x) \leqq \underline{d}_{v}(x)$ for almost every $x \in F_{C, \alpha}$. The result follows since $F=\bigcup_{C, \alpha} F_{C, \alpha}$.

The last statement is a direct consequence of the preceding statements.

Acknowledgement. The authors would like to thank H. Furstenberg, F. Ledrappier, A. Manning, and Y. Peres for helpful discussions. We would like to especially thank L. Barreira for his fruitful comments and examples.

In a previous version of this manuscript we studied geometric constructions with disjoint basic sets. We did not consider the weaker open-set condition and we wish to thank D. Mauldin, whose remarks helped us to better understand the role of this condition in the definition of Moran-like geometric constructions. It was a simple observation that all our arguments carried through under an assumption which is even weaker than the open-set condition. We were amazed at the sizable literature (references provided by Mauldin) where authors attempted to extend dimension results to constructions with non-overlapping basic sets versus disjoint basic sets. In view of our paper, this appears to be an inconsequential distinction.

The first author wishes to thank IHES for their support and hospitality during his visit in the Fall 1992 which enabled him to think about some problems in this paper.

\section{Appendices}

\section{Appendix 1. Hausdorff Dimension and Box Dimension}

Let $U \subset \mathbb{R}^{n}$. The diameter of $U$ is defined as $\operatorname{diam}(U)=\sup \{|x-y|: x, y \in U\}$. If $\left\{U_{i}\right\}$ is a countable collection of sets of diameter at most $\delta$ that cover $Z$, i.e., $Z \subset \bigcup_{i} U_{l}$ with $0<\left|U_{i}\right| \leqq \delta$ for each $i$, we say that $\left\{U_{i}\right\}$ is a $\delta$-cover of $Z$.

Suppose that $Z \subset \mathbb{R}^{n}$ and $s \geqq 0$. For any $s>0$ we define

$$
m_{H}(s, Z)=\lim _{\delta \rightarrow 0} \inf _{\left\{U_{l}\right\}}\left\{\sum_{i} \operatorname{diam}\left(U_{i}\right)^{s}:\left\{U_{l}\right\} \text { is a } \delta \text {-cover of } Z\right\} .
$$

We call $m_{H}(s, Z)$ the $s$-dimensional Hausdorff measure of $Z$. There exists a unique critical value of $s$ at which $m_{H}(s, Z)$ jumps from $\infty$ to 0 . This critical value is called the Hausdorff dimension of $Z$ and is written $\operatorname{dim}_{H} Z$. If $s=\operatorname{dim}_{H} Z$, then $m_{H}(s, Z)$ may be $0, \infty$, or finite. Hence $\operatorname{dim}_{H} Z=\sup \left\{s: m_{H}(s, Z)=\infty\right\}=\inf \{s:$ $\left.m_{H}(s, Z)=0\right\}$.

Let $N_{\delta}(Z)$ denote the minimum number of sets of diameter precisely $\delta$ needed to cover the set $Z$. We define the lower and upper box dimensions of $Z$ by

$$
\underline{\operatorname{dim}}_{B} Z=\liminf _{\delta \rightarrow 0} \frac{\log N_{\delta}(Z)}{\log \left(\frac{1}{\delta}\right)} \text { and } \overline{\operatorname{dim}}_{B} Z=\limsup _{\delta \rightarrow 0} \frac{\log N_{\delta}(Z)}{\log \left(\frac{1}{\delta}\right)} .
$$

It is easy to see that $\operatorname{dim}_{H} Z \leqq \underline{\operatorname{dim}}_{B} Z \leqq \overline{\operatorname{dim}}_{B} Z$. 


\section{Appendix 2. Two Methods of Obtaining Lower Bounds for $\operatorname{dim}_{H} F$}

Uniform Mass Distribution Principle [Fr]. Let $\mu$ be a probability measure supported on of $Z \subset \mathbb{R}^{n}$ and suppose that for some $s$ there are numbers $c>0$, and $\delta>0$ such that $\mu(U) \leqq c \operatorname{diam}(U)^{s}$ for all sets $U \subset Z$ with $\operatorname{diam}(U)^{s} \leqq \delta$. Then $m_{H}(s, Z) \geqq \mu(Z) / c$ and $s \leqq \operatorname{dim}_{H} Z$.

Non-Uniform Mass Distribution Principle. Let $\mu$ be a probability measure supported on $Z \subset \mathbb{R}^{n}$. We define the Hausdorff dimension of the measure $\mu, \operatorname{dim}_{H} \mu=$ $\inf \left\{\operatorname{dim}_{H} U, \mu(U)=1\right\}$, and the lower and upper pointwise dimensions of $\mu$, $\underline{d}_{\mu}(x)=\liminf _{\varepsilon \rightarrow 0} \frac{\log \mu(B(x, \varepsilon))}{\log \varepsilon}$ and $\bar{d}_{\mu}(x)=\limsup _{\varepsilon \rightarrow 0} \frac{\log \mu(B(x, \varepsilon))}{\log \varepsilon}$.

Proposition. If $\underline{d}_{\mu}(x) \geqq d \geqq 0$ for a.e. $x \in Z$, then $\operatorname{dim}_{H} \mu \geqq d$, and hence $\operatorname{dim}_{H} Z$ $\geqq d$.

The uniform mass distribution principle implies the non-uniform mass distribution principle. In fact, $d_{\mu}^{L}(x) \geqq d$ implies that for any $\alpha>0, \mu(B(x, r)) \leqq$ $C(x, \alpha) r^{d-\alpha}$.

\section{Appendix 3. Thermodynamic Formalism}

Good references for this material are [Bo1] and [PP]. Given a transitive $p \times p$ transfer matrix $A$ of $0 s$ and $1 s$, consider the one-sided subshift of finite type $\left(\Sigma_{A}^{+}, \sigma\right)$ with $\sigma: \Sigma_{A}^{+} \rightarrow \Sigma_{A}^{+}$the shift map. The set $\Sigma_{A}^{+}$consists of all admissible words, i.e., a word $x=\left(x_{1}, x_{2}, \ldots\right) \in\{1, \ldots, p\}^{\mathbb{N}}$ is admissible if $a_{x_{1}, x_{1+1}}=1$ for all $i \in \mathbb{N}$. The space $\Sigma_{A}^{+}$has a natural family of metrics defined by $d(x, y)=\sum_{k=1}^{\infty} \frac{\left|x_{k}-y_{k}\right|}{\beta^{k}}$, where $\beta$ is any number satisfying $\beta>1$. The set $\Sigma_{A}^{+}$is compact with respect to the topology induced by $d$ and the shift map $\sigma: \Sigma_{A}^{+} \rightarrow \Sigma_{A}^{+}$is a homeomorphism. We let $\Sigma_{p}^{+}=\{1, \ldots, p\}^{\mathbb{N}}$.

More generally, we consider a general symbolic system, i.e., a compact subset $Q \subset \Sigma_{p}^{+}$that is $\sigma$-invariant, i.e., $\sigma(Q)=Q$.

Let $C^{0}(Q), C^{\alpha}(Q)$ denote the spaces of continuous and $\alpha$-Hölder continuous functions on $Q$. We define a mapping $P: C^{0}(Q) \rightarrow \mathbb{R}$ by

$$
P(\phi)=\lim _{n \rightarrow \infty} \frac{1}{n} \log \left(\sum_{\substack{\left(l_{1} \cdots i_{n}\right) \\ \text { admissible }}} \inf _{\left.x \in \Delta_{\left(l_{1}\right.} i_{n}\right)} \exp \left(S_{n} \phi(x)\right)\right),
$$

where $S_{n} \phi(x)=\sum_{i=0}^{n-1} \phi\left(\sigma^{l} x\right)$. We call $P(\phi)$ the topological pressure of $\phi$.

The following result is a variational characterization of pressure that is valid for all topological dynamical systems. Let $\mathfrak{M}(Q)$ denote the space of all shift-invariant Borel probability measures on $Q$.

Variational Principle. Let $\phi \in C^{0}(Q)$. Then

$$
P(\phi)=\sup _{\mu \in \mathfrak{M}(Q)}\left(h_{\mu}(\sigma)+\int_{Q} \phi d \mu\right) \text {. }
$$


Measures that realize the variational principle for topological pressure play crucial roles in the thermodynamical formalism.

Definition. A Borel probability measure $\mu=\mu_{\phi}$ on $Q$ is called an equilibrium measure for the potential $\phi$ if

$$
P(\phi)=h_{\mu}(\sigma)+\int_{\Sigma_{A}} \phi d \mu .
$$

Since the shift map on a general symbolic system is expansive, the supremum in the variational principle is attained by some measure [W]. This measure need not be unique.

Another important class of measures are Gibbs measures:

Definition. Let $\phi \in C^{0}(Q)$. A Borel probability measure $\mu=\mu_{\phi}$ on $Q$ is called a Gibbs measure for the potential $\phi$ if there exist constants $D_{1}, D_{2}>0$ such that

$$
D_{1} \leqq \frac{\mu\left\{y: y_{i}=x_{i}, i=0, \ldots, n-1\right\}}{\exp \left(-n P(\phi)+\sum_{k=0}^{n-1} \phi\left(\sigma^{k} x\right)\right)} \leqq D_{2}
$$

for all $x=\left(x_{1} x_{2} \cdots\right) \in \Sigma_{A}^{+}$and $n \geqq 0$.

For subshifts of finite type, Gibbs measures exist for any Holdër continuous potential $\phi$, are unique, and coincide with the equilibrium measure for $\phi$. Little is known about the existence of Gibbs measures for general symbolic systems.

The main tool used in constructing and studying Gibbs measures for subshifts of finite type is the (linear bounded) transfer operator $L_{f}: C^{\alpha}\left(\Sigma_{A}^{+}\right) \rightarrow C^{\alpha}\left(\Sigma_{A}^{+}\right)$defined by

$$
\left(L_{f} \phi\right)(x)=\sum_{y \in \sigma^{-1}(x)} \exp (f(y)) \phi(y)=\sum_{k} \exp (f(k x)) \phi(k x)
$$

along with its dual operator $L_{f}^{*}: M\left(\Sigma_{A}^{+}\right) \rightarrow M\left(\Sigma_{A}^{+}\right)$, where $M\left(\Sigma_{A}^{+}\right)$denotes the space of Borel measures on $\Sigma_{A}^{+}$. The following theorem of Ruelle constructs Gibbs measures using the operator $L_{f}$ :

Proposition [PP]. Let $\left(\Sigma_{A}^{+}, \sigma\right)$ be a mixing subshift of finite type. There exists a number $\lambda=\exp (P(\phi)), h \in C^{0}\left(\Sigma_{A}^{+}, \mathbb{R}\right)$ with $h>0$ and $v \in M\left(\Sigma_{A}^{+}\right)$for which $L_{f} h=\lambda h, L_{f}^{*} v=\lambda v$, and $v(h)=1$. Then $\mu=h v$ is a $\sigma$-invariant probability measure on $\Sigma_{A}^{+}$and is a Gibbs measure for $\phi$.

In this paper we deal exclusively with a special class of potentials that depend only on the first coordinate, i.e., $\phi(x)=\phi\left(x_{1}\right)$. In this case, the measures $v$ and $\mu$ are Markov and can be described explicitly: the eigenfunction $h=h\left(x_{1}\right)$ satisfies

$$
\sum_{i} h(i) A(i, j) \exp (\phi(i))=\lambda h(j),
$$

and the measure $v$ is defined on cylinder sets by

$$
v\left[i_{0}, \ldots, i_{n}\right]=P\left(i_{0}, i_{1}\right) \cdots P\left(i_{n-1}, i_{n}\right) p\left(i_{n}\right) \quad \text { where } P(i, j)=\frac{A(i, j) h(i) \exp (\phi(i))}{\lambda h(j)}
$$


and $P p=p$ with $\sum_{i} p(i)=1$. Finally, the Gibbs measure $\mu$ is defined by $d \mu=h\left(x_{1}\right) d v$.

The Gibbs measure $\mu$ is unique provided the potential $f$ is Hölder continuous.

Proposition [PP]. Given numbers $\lambda_{i}, i=1, \ldots, p$, define the function

$$
\phi: \Sigma_{A}^{+} \rightarrow \Sigma_{A}^{+} \quad \text { by } \phi(x)=\phi\left(x_{1}, x_{2}, \ldots\right)=\log \lambda_{x_{1}} .
$$

Then $\phi$ is Hölder continuous with respect to the standard metric. Furthermore, there exists a unique $s \geqq 0$ such that $P(s \phi)=0$.

\section{Appendix 4. Gauge Functions}

One can generalize the definition of Hausdorff measure to give more refined information about a set whose Hausdorff measure at the dimension is zero.

Suppose $h(t)$ is a continuous increasing function defined on $(0, \varepsilon)$ with $\varepsilon>0$ such that $h(t) \rightarrow 0$ as $t \rightarrow 0$. Using the notation in Appendix 1, we define

$$
\left.m_{H}^{h}(Z) \stackrel{\text { def }}{\equiv} \lim _{\delta \rightarrow 0} \inf _{\left\{U_{l}\right\}}\left\{\sum_{i} h\left(\operatorname{diam}\left(U_{i}\right)\right):\left\{U_{i}\right\} \text { is a } \delta \text {-cover of } Z\right\}\right\} .
$$

We call $m_{H}^{h}(Z)$ the $s$-dimensional Hausdorff measure of $Z$ with respect to the gauge function $h$. Clearly letting $h(t)=t^{s}$ gives the $s$-dimensional Hausdorff measure defined in Appendix 1.

\section{Appendix 5. Expanding Maps}

A continuous map $g: X \rightarrow X$ of a compact metric space $X$ is expanding if $g$ is a local homeomorphism and there exist constants $F \geqq E>1$ and $r_{0}>0$ such that

$$
B(g(x), E r) \subset g(B(x, r)) \subset B(g(x), F r)
$$

for every $x \in X$ and $0<r<r_{0}$.

Without loss of generality we may assume that for any $x \in X$, the map $g$ restricted to the ball $B\left(x, r_{0}\right)$ is a homeomorphism.

We recall that a Markov partition for an expanding map $g$ is a finite cover of $X$ by elements (called rectangles) $\left\{R_{1}, \ldots, R_{p}\right\}$ such that:

(1) each rectangle $R_{i}$ is the closure of its interior int $R_{i}$;

(2) int $R_{i} \cap \operatorname{int} R_{j}=\emptyset$ unless $i=j$;

(3) each $g\left(R_{i}\right)$ is a union of rectangles $R_{j}$.

The Markov partition generates a symbolic model of the map $g$ by a subshift of finite type $\left(\Sigma_{A}^{+}, \sigma\right)$ where $A=\left(a_{i j}\right)$ is the transfer matrix of the Markov partition, i.e., $a_{i j}=1$ if int $R_{i} \cap g^{-1}$ (int $\left.R_{j}\right) \neq \emptyset$ and $a_{i j}=0$ otherwise. This gives a coding map $\chi: \Sigma_{A}^{+} \rightarrow X$ such that

$$
\chi(\omega)=\bigcap_{n \geqq 0} g^{-n}\left(R_{i_{n}}\right), \quad \omega=\left(i_{1} i_{2} \cdots\right),
$$


and the following diagram

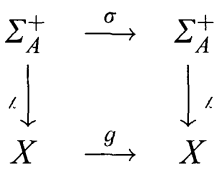

is commutative. Under the coding map the cylinder sets $C_{i_{1} \cdots i_{n}} \in \Sigma_{A}^{+}$correspond to the basic sets in $X$ generated by the Markov partition

$$
R_{i_{1} \cdots i_{n}}=R_{i_{1}} \cap g^{-1} R_{i_{2}} \cap \cdots \cap g^{-n} R_{i_{n}} .
$$

The map $\chi$ is Hölder continuous and injective on the set of points whose trajectories never hit the boundary of any element of the Markov partition. The pullback by $\chi$ of any Hölder continuous function on $X$ is a Hölder continuous function on $\Sigma_{A}^{+}$.

There is a special Markov partition for an expanding map such that the rectangle containing a given point in $X$ is almost a ball ([PW2]).

We say that a continuous expanding map $g$ on a compact metric space $X$ is conformal if there exist a continuous function $a(x)$ with $|a(x)|>1$ on $X$ and positive constants $C_{1}, C_{2}$, and $r_{0}$ such that for any $0<r \leqq r_{0}$, any two points $x, y \in X$, and any integer $n \geqq 0$ we have: if $\rho\left(g^{k}(x), g^{k}(y)\right) \leqq r_{0}$ for all $k=0,1, \ldots n$, then

$$
C_{1} \prod_{k=0}^{n}\left|a\left(g^{k}(x)\right)\right|^{-1} \leqq \rho\left(g^{n}(x), g^{n}(y)\right) \leqq C_{2} \prod_{k=0}^{n}\left|a\left(g^{k}(x)\right)\right|^{-1} \text {. }
$$

\section{Appendix 6. Axiom $\mathbf{A}^{\#}$ Homeomorphisms}

Let $(X, \rho)$ be a compact metric space with metric $\rho$ and $f: X \rightarrow X$ a homeomorphism. Given $x \in X$ and $\varepsilon>0$, we define the local stable and unstable sets at $x$ by

$$
\begin{aligned}
& W_{\varepsilon}^{(s)}=\left\{y \in X: \rho\left(f^{n}(x), f^{n}(y)\right) \leqq \varepsilon \text { for all } n \geqq 0\right\}, \\
& W_{\varepsilon}^{(u)}=\left\{y \in X: \rho\left(f^{-n}(x), f^{-n}(y)\right) \leqq \varepsilon \text { for all } n \geqq 0\right\} .
\end{aligned}
$$

Following Bowen [Bol] and Alekseyev and Jakobson [AJ], we say that $f$ is an Axiom $A^{\#}$ homeomorphism if there exist constants $0<\lambda<1$ and $\varepsilon>0$ such that

(a) for each $x \in X$,

$$
\begin{aligned}
& \rho\left(f^{n}(y), f^{n}(z)\right) \leqq \lambda^{n} \rho(y, z) \quad \text { for all } y, z \in W^{(s)}(x) \text { and } n \geqq 0 \text {, } \\
& \rho\left(f^{-n}(y), f^{-n}(z)\right) \leqq \lambda^{n} \rho(y, z) \quad \text { for all } y, z \in W^{(s)}(x) \text { and } n \geqq 0 \text {; }
\end{aligned}
$$

(b) there exists $\delta>0$ such that for all $x, y \in X$ with $\rho(x, y)<\delta$ the intersection $W_{\varepsilon}^{(s)}(x) \cap W_{\varepsilon}^{(u)}(y)$ consists of a single point which we denote by $[x, y]$;

(c) the map

$$
[\cdot, \cdot]:\{(x, y) \in X \times X: \rho(x, y)<\delta\} \mapsto X
$$

is continuous.

We recall some facts about Axiom $A^{\#}$ homeomorphisms (see [Bow1], [AY]). A point $x \in X$ is called non-wandering if for each neighborhood $U$ of $x$ there exists $n \geqq 0$ such that $f^{n}(U) \cap U \neq \emptyset$. We denote by $\Omega(f)$ the set of non-wandering 
points of $f$. This set is $f$-invariant, closed, and is the closure of the set of periodic points of $f$. It also admits a decomposition into a finite number of disjoint closed $f$-invariant sets (called basic sets), $\Omega(f)=\Lambda_{1} \cup \cdots \cup \Lambda_{n}$, such that $f \mid \Lambda_{i}$ is topologically transitive.

We call a non-empty set $R$ a rectangle if $\operatorname{diam} R \leqq \delta, R$ is the closure of its interior, and $[x, y] \in R$ whenever $x, y \in R$. For each $x \in R$ we write $W^{(s)}(x, R)=$ $W_{\varepsilon}^{(s)}(x) \cap R$ and $W^{(u)}(x, R)=W_{\varepsilon}^{(u)}(x) \cap R$. Let $\Lambda$ be a closed $f$-invariant set. A finite cover $\left\{R_{1}, \ldots, R_{p}\right\}$ of $\Lambda$ by a rectangles is called a Markov partition if

(1) $R_{\imath} \cap R_{j} \subset \partial R_{i} \cap \partial R_{j}$ for $i \neq j$;

(2) for each $x \in$ int $R_{i} \cap f^{-1}\left(\right.$ int $R_{j}$ ) we have

$$
f\left(W^{(u)}\left(x, R_{i}\right)\right) \supset W^{(u)}\left(f(x), R_{J}\right), \quad f\left(W^{(s)}\left(x, R_{i}\right)\right) \subset W^{(s)}\left(f(x), R_{j}\right) .
$$

The Markov partition $\left\{R_{1}, \ldots, R_{p}\right\}$ generates a symbolic model of the map $f$ by a subshift of finite type $\left(\Sigma_{A}, \sigma\right)$. Namely, we define the $p \times p$ transfer matrix $A=\left(a_{i j}\right)$ by setting $a_{i j}=1$ if $\operatorname{int} R_{i} \cap f^{-1}$ (int $\left.R_{j}\right) \neq \emptyset$ and $a_{i j}=0$ otherwise. Then $\Sigma_{A}$ is the set of two-sided sequences $\omega=\left(i_{n}\right)_{n \in \mathbb{Z}}$ which are admissible with respect to $A$, i.e., $a_{i_{n} i_{n+1}}=1$ for all $n \in \mathbb{Z} ; \sigma$ is the shift. For each $\omega=\left(i_{n}\right) \in \Sigma_{A}$ and $n \geqq 0$ we set

$$
\chi(\omega)=\bigcap_{n=-\infty}^{\infty} f^{-n}\left(R_{i_{n}}\right) .
$$

One can show that $\chi(\omega)$ consists of a single point. Thus, we obtain a coding map $\chi: \Sigma_{A} \rightarrow \Lambda$ which is continuous and onto. Moreover, the following diagram

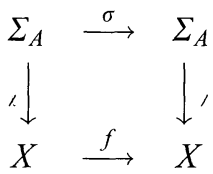

is commutative.

There is a special Markov partition for an Axiom $A^{\#}$ homomorphism on a basic set $\Lambda$ such that the rectangle containing a given point $x$ in $\Lambda$ is almost a ball (see [W1]).

An Axiom $A^{\#}$-homeomorphism is called conformal if

the maps $f \mid W_{\varepsilon}^{(u)}(x)$ and $f^{-1} \mid W_{\varepsilon}^{(s)}(x)$ are conformal and expanding.

\section{References}

[AJ] Alekseyev, V., Jakobson, M.: Symbolic Dynamics and Hyperbolic Dynamical Systems. Phys. Rep. 75, 287-325 (1981)

[AS] Afraimovich, V., Shereshevsky, M.: The Hausdorff Dimension of Attractors Appearing by Saddle-Node Bifurcations. Int. J. Bifurcation and Chaos 1:2, 309-325 (1991)

[Ba] Barreira, L.: Cantor Sets with Complicated Geometry and Modeled by General Symbolic Dynamics. To appear, Random and Computational Dynamics (1995)

[BFKO] Bourgain, J.: Pointwise Ergodic Theorems for Arithmetic Sets, with an Appendix by J. Bourgain, H. Furstenberg, Y. Katznelson, and D. Ornstein Publ. Math. IHES 69, 5-45 (1989)

[BK] Brin, M., Katok, A.: On Local Entropy. Lecture Notes in Mathematics 1007, Geometric Dynamics (1981), Berlin-Heidelberg-New York, Springer Verlag, pp. 30-38 
[Bo1] Bowen, R.: Equilibrium States and the Ergodic Theory of Anosov Diffeomorphisms. Springer Lecture Notes \#470, Berlin-Heidelberg-New York, Springer Verlag, 1975

[Bo2] BowBo, R.: Hausdorff Dimension of Quasi-circles. Publ. Math. IHES 50, 11-25 (1979)

[BM] Bertrand-Mathis, A.: Questions Diverses Relatives aux Systèms codés: Applications au $\theta$-shift. Preprint

[BU] Bedford, T., Urbański, M.: The box and Hausdorff Dimension of Self-Affine Sets. Ergod. Th. and Dynam. Systems 10, 627-644 (1990)

[C] Cutler, C.D.: Connecting Ergodicity and Dimension in Dynamical Systems. Ergod. Th. and Dynam. Systems 10, 451-462 (1990)

[CLP] Collet, P., Lebowitz, J.L., Porzio, A.: The Dimension Spectrum of Some Dynamical Systems. J. Stat. Physics 47, 609-644 (1987)

[CM] Cawley, R., Mauldin, R.: Adv. Math. 92, 196-236 (1992)

[EM] Mauldin, R., Edgar, G.: Multifractal Decomposition of Digraph Recursive fractals. Proc. London Math. Soc. 65, 604-628 (1992)

[ER] Eckmann, J.P., Ruelle, D.: Ergodic Theory of Chaos and Strange Attractors. 3. Rev. Mod. Phys. 57, 617-656 (1985)

[F1] Falconer, K.: Fractal Geometry, Mathematical Foundations and Applications. Cambridge: Cambridge Univ. Press, 1990

[F2] Falconer, K.: Random Fractals. Math. Proc. Camb. Phil. Soc. 100, 559-582 (1986)

[Fe] Federer, H.: Geometric Measure Theory. Berlin-Heidelberg-New York: Springer Verlag, 1969

[Fr] Frostman, O.: Potential d'équilibre et Capacité des Ensembles Avec Quelques Applications à la Théorie des Fonctions. Meddel. Lunds Univ. Math. Sem. 3, 1-118 (1935)

[Fu] Furstenberg, H.: Disjointness in Ergodic Theory, Minimal Sets, and a Problem in Diophantine Approximation. Mathematical Systems Theory 1, 1-49 (1967)

[G] Graf, S.: Statistically Self-similar Fractals. Prob. Theory and Related Fields 74, 357-397 (1987)

[GMW] Graf, S., Mauldin, D., Williams, S.: The Exact Hausdorff Dimension in Random Recursive Constructions. 381, Mem. Am. Math. Soc. 71, (1988)

$[\mathrm{KH}] \quad$ Katok, A., Hasselblatt, B.: Introduction to the Modern Theory of Dynamical Systems. Cambridge: Cambridge University Press, 1995

[HP] Hentschel, H.G.E., Procaccia, I.: The Infinite Number of Generalized Dimensions of Fractals and Strange Attractors. Physica 8D, 435-444 (1983)

[K] Kahane, J.P.: Sur le Modéle de Turbulence de Benoit Mandelbrot. C.R. Acad. Sci. Paris 278A, 621-623 (1974)

[L] Ledrappier, F.: Dimension of Invariant Measures. Preprint (1992)

[Lo] Lopes, A.: The Dimension Spectrum of the Maximal Measure. SIAM J. Math. Analysis 20, 1243-1254 (1989)

[LM] Ledrappier, F., Misiurewicz, M.: Dimension of Invariant Measures for Maps with Exponent Zero. Ergod. Th. and Dynam. Systems 5, 595-610 (1985)

[LY] Ledrappier, F., Young, L.S.: The Metric Entropy of Diffeomorphisms. Part II. Ann. of Math. 122, 540-574 (1985)

[Mc] McMullen, C.: The Hausdorff Dimension of General Sierpiński Carpets. Nagoya Math. J. 96, 1-9 (1984)

[MW1] Mauldin, R., Williams, S.: Hausdorff Dimension in Graph Directed Constructions. Trans. AMS 309:2, 811-829 (1988)

[MW2] Mauldin, R., Williams, S.: Random Geometric Constructions: Asymptotic, Geometric and Asymptotic Properties. Trans. Am. Math. Soc. 295, 325-346 (1986)

[Mo] Moran, P.: Additive Functions of Intervals and Hausdorff Dimension. Proc. Camb. Phil. Society 42, 15-23 (1946)

[OSY] Ott, E., Sauer, T., Yorke, J.: Lyapunov Partition Functions for the Dimensions of Chaotic Sets. no 9, Phys. Rev. A 39, 4212-4222 (1989)

[PP] Parry, W., Pollicott, W.: Zeta Functions and the Periodic Orbit Structures of Hyperbolic Dynamics. Astérisque 187-188, (1990)

[PW1] Pesin, Y., Weiss, H.: On the Dimension of Deterministic and Random Cantor-like Sets. Math. Res. Lett. 1, 519-529 (1994)

[PW2] Pesin, Y., Weiss, H.: A Multifractal Analysis of Equilibrium Measures for Conformal Expanding Maps and Markov Moran Geometric Constructions. Preprint (1995) 
[PoW] Pollicott, M., Weiss, H.: The Dimensions of Some Self-affine Limit Sets in the Plane and Hyperbolic Sets. Preprint (1993)

[PU] Przytycki, F., Urbański, M.: On Hausdorff Dimension of Some Fractal Sets. Studia Mathematica 93, 155-186 (1989)

[PY] Pesin, Y., Yue, C.B.: Hausdorff Dimension of Measures with Non-zero Lyapunov Exponents and Local Product Structure. PSU Preprint (1993)

[R] Ruelle, D.: Thermodynamic Formalism. Reading, Addison-Wesley, 1978

[S] Shereshevsky, M.: On the Hausdorff Dimension of a Class of Non-Self-Similar Fractals. Math. Notes 50, no. 5, 1184-1187 1991

[Sh] Shub, M.: Global Stability of Dynamical Systems. Berline-Heidelberg-New York: Springer Verlag, 1987

[St] Stella, S.: On Hausdorff Dimension of Recurrent Net Fractals. Proc. Am. Math. Soc. 116, 389-400 (1992)

[Y] Young, L.S.: Dimension, Entropy, and Lyapunov Exponents. Ergod. Th. and Dynam. Systems 2, 109-124 (1982)

[W] Walters, P.: Introduction to Ergodic Theory. Berlin-Heidelberg-New York: Springer Verlag, 1982

[We] Weiss, B.: Subshifts of Finite Type and Sophic Systems. Monatschefte für Mathematik 77, 462-474 (1973)

[W1] Weiss, H.: The Multifractal Analysis of Topologically Hyperbolic Homeomorphisms. In preparation

Communicated by Ya.G. Sinai 
